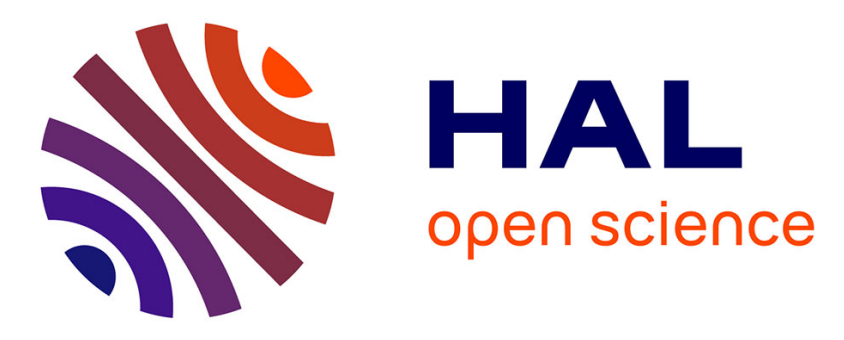

\title{
Perturbed DNA methylation by sustained overexpression of Gadd45b induces chromatin disorganization, DNA strand breaks and dopaminergic neuron death in mice
}

Rajiv L Joshi, Camille Ravel-Godreuil, Olivia Massiani-Beaudoin, Philippe Mailly, Alain Prochiantz, Julia Fuchs

\section{To cite this version:}

Rajiv L Joshi, Camille Ravel-Godreuil, Olivia Massiani-Beaudoin, Philippe Mailly, Alain Prochiantz, et al.. Perturbed DNA methylation by sustained overexpression of Gadd45b induces chromatin disorganization, DNA strand breaks and dopaminergic neuron death in mice. 2020. hal-03066810

\author{
HAL Id: hal-03066810 \\ https://hal.science/hal-03066810
}

Preprint submitted on 18 Dec 2020

HAL is a multi-disciplinary open access archive for the deposit and dissemination of scientific research documents, whether they are published or not. The documents may come from teaching and research institutions in France or abroad, or from public or private research centers.
L'archive ouverte pluridisciplinaire HAL, est destinée au dépôt et à la diffusion de documents scientifiques de niveau recherche, publiés ou non, émanant des établissements d'enseignement et de recherche français ou étrangers, des laboratoires publics ou privés. 
1 Title:

2

3 Perturbed DNA methylation by sustained overexpression of Gadd45b induces

4 chromatin disorganization, DNA strand breaks and dopaminergic neuron death 5 in mice

8 Authors:

9 Camille Ravel-Godreuil ${ }^{1}$, Olivia Massiani-Beaudoin ${ }^{1}$, Philippe Mailly ${ }^{2}$, Alain 10 Prochiantz ${ }^{1}$, Rajiv L. Joshi ${ }^{1^{*}}$ and Julia Fuchs ${ }^{1^{*}}$

Affiliations:

$14{ }^{1}$ Centre for Interdisciplinary Research in Biology (CIRB), Collège de France, INSERM 15 U1050/ CNRS UMR 7241, PSL Research University, 11 place Marcelin Berthelot, 75231 Paris Cedex 05, France.

${ }^{2}$ Orion Imaging Facility, Center for Interdisciplinary Research in Biology (CIRB), College de France, CNRS Unité Mixte de Recherche 724, INSERM Unité 1050,

$22{ }^{*}$ For correspondence:

23 J.F. (Julia.fuchs@college-de-france.fr); R.L.J. (rajiv.joshi@college-de-france.fr) 


\section{Abstract}

Heterochromatin disorganization is a key hallmark of aging and DNA methylation state is currently the main molecular predictor of chronological age. The most frequent neurodegenerative diseases like Parkinson disease and Alzheimer's disease are age-related but how the aging process and chromatin alterations are linked to neurodegeneration is unknown. Here, we investigated the consequences of viral overexpression of Gadd45b, a multifactorial protein involved in active DNA demethylation, in the midbrain of wild-type mice. Gadd45b overexpression induces global and stable changes in DNA methylation, particularly on gene bodies of genes related to neuronal functions. DNA methylation changes were accompanied by perturbed H3K9me3-marked heterochromatin and increased DNA damage. Prolonged Gadd45b expression resulted in dopaminergic neuron degeneration accompanied by altered expression of candidate genes related to heterochromatin maintenance, DNA methylation or Parkinson disease. Gadd45b overexpression rendered midbrain dopaminergic neurons more vulnerable to acute oxidative stress. Heterochromatin disorganization and DNA demethylation resulted in derepression of mostly young LINE-1 transposable elements, a potential source of DNA damage, prior to Gadd45b-induced neurodegeneration. Our data implicate that alterations in DNA methylation and heterochromatin organization, LINE-1 derepression and DNA damage can represent important contributors in the pathogenic mechanisms of dopaminergic neuron degeneration with potential implications for Parkinson disease. 


\section{Introduction}

Epigenetic marks separate chromatin into actively transcribed euchromatin and repressive heterochromatin domains, and participate in the spatial organization of the genome into highly structured $3 D$ domains ${ }^{1}$. These epigenetic signatures include DNA methylation, various post-translational modifications of histones, and attraction forces between different types of genomic repeat elements, including transposable elements (TEs) ${ }^{1}$. Changes in chromatin structure regulate DNA accessibility to transcription factors and the physical proximity of enhancers to promoters, thereby regulating gene expression.

It is well established that DNA methylation regulates various important cellular processes during development and cell differentiation. In recent years, however, perturbations of chromatin organization have been linked to the aging process and global changes in DNA methylation are currently the main molecular predictor of chronological age (reviewed in ${ }^{2}$ ). Aging-induced epigenetic remodeling of chromatin can impact on genomic stability ${ }^{3,4}$, and vice versa ${ }^{2}$. Thus chromatin states and genomic stability are important, interdependent factors associated with the aging process ${ }^{5}$. One emerging culprit related to both processes is the un-silencing of transposable elements (TEs) with age. Around half of the human genome is comprised of TEs. The evolutionary most successful TEs in mammals are long interspersed nuclear element-1 (LINE-1 or L1). Mostly fossilized and a few remaining active LINE-1 sequences (around 100 in humans and 3000 in mice) represent around $17 \%$ of the human ${ }^{6}$ and $21 \%$ of the mouse genome ${ }^{7}$. Young and full-length LINE-1 elements are autonomous retrotransposons, expanding in the genome through a "copy and paste" retrotransposition mechanism and encoding the two necessary proteins, namely ORF $1 p$ and ORF $2 p$, required for their mobilization. ORF1p is an RNA binding protein with strong "cis" preference ${ }^{8-10}$ and ORF2p encodes an endonuclease and a reverse transcriptase ${ }^{11,12}$. Several repressive cellular mechanisms, including DNA methylation and heterochromatinization, limit their expression ${ }^{13}$. When these fail with age, TEs can become derepressed ${ }^{14}$. An increased activity of LINE-1 is associated with genomic instability through the induction of DNA damage ${ }^{15-18}$.

How aging and neurodegeneration are linked at the molecular level remains widely unknown. This question is, however, of high relevance as age is the primary 
83 risk factor for the most common neurodegenerative diseases (NDs) including 84 Parkinson disease (PD) and Alzheimer disease (AD) ${ }^{19}$. Some of the cellular 85 processes defined as the hallmarks of aging ${ }^{20}$ overlap with pathways shown to be dysfunctional in NDs. This is the case for impaired proteostasis, mitochondrial dysfunction, deregulated nutrient sensing, increased oxidative stress and neuroinflammation ${ }^{21,22}$. Whether two other important nominators of aging, namely perturbations in the organization of chromatin and genomic instability, are associated with neuronal aging and neurodegeneration is not unequivocally proven yet.

A cardinal feature of PD is the degeneration of midbrain dopaminergic (mDA) neurons in the substantia nigra pars compacta (SNpc). These neurons project to the striatum and their loss leads to a striatal dopamine deficiency inducing the typical motor symptoms of PD. Decreased global DNA methylation with age in the SNpc has been observed ${ }^{23}$ and DNA methylation changes, mostly on specific genetic risk loci, have been linked to several NDs ${ }^{24}$ including $P D{ }^{25}$. Alterations in histone modifications have also been observed in PD ${ }^{26}$. However, the possible contribution of age-related epigenetic alterations to the pathogenesis of PD and the onset of neurodegeneration has not been demonstrated yet.

Here, we investigated how SNpc mDA neurons react to perturbations of chromatin organization. For this purpose, we overexpressed Gadd45b in the SNpc of wild-type mice using an adeno-associated virus (AAV) vector. GADD45B is a multifunctional protein which coordinates in the nucleus an active DNA demethylation pathway involving cytidine deaminases and DNA glycosylases ${ }^{27}$ in association with the base excision repair (BER) pathway ${ }^{28}$. We chose GADD45B because it is a known DNA demethylase in postmitotic neurons ${ }^{27,29}$ and it is highly inducible in conditions of oxidative stress in the SNpc ${ }^{30}$. We show that overexpression of Gadd45b in the SNpc of wild-type mice leads to widespread perturbations of DNA methylation, heterochromatin disorganization, increased vulnerability to oxidative stress of mDA neurons, activation of LINE-1 elements, DNA strand breaks and neuronal death. Our data reinforces the hypothesis that aging-induced global chromatin disorganization initiates neurodegeneration, possibly via the derepression of LINE-1 elements. 


\section{Results}

Gadd45b overexpression in the SNpc of wild-type mice leads to early and stable DNA methylation perturbations in gene bodies of genes related to neuronal functions

Wild-type mice littermates (6 weeks old) were injected unilaterally in the SNpc using AAV8-mCherry or AAV8-mGadd45b. The animals were sacrificed after 14 or 90 days post-injectionem (p.i.) and either perfused or dissected as schematized in Figure 1A. The AAV8-mCherry control virus efficiently infected tyrosine hydroxylase positive $(\mathrm{TH}+)$ neurons of the SNpc as shown by mCherry expression at 14d p.i. (Fig. 1B, upper panel). Due to the lack of a good antibody against GADD45B, expression of Gadd45b was verified at 14d p.i. by in situ hybridization (Fig1. B, lower panel) and RT-qPCR after manual micro-dissection of the SNpc at $14 \mathrm{~d}$ and $90 \mathrm{~d}$ p.i. (Fig. 1C). Gadd45b transcripts on the injected ipsilateral side were increased up to 79-fold on average $(11.02 \pm 4.83 ; 869.1 \pm 283 ; n=6)$ at 14 d p.i. and up to 178 -fold $(7.89 \pm 1.52$; $1402 \pm 683 ; n=4)$ at 90 d p.i. compared to the endogenous transcript levels of the noninjected contralateral side.

Having verified the efficient overexpression of Gadd45b in the SNpc, we asked whether this perturbs DNA methylation patterns in the SNpc. To this end, we injected wild-type mice with AAV8-mGadd45b $(n=6)$ or AAV8-mCherry $(n=6)$ and simultaneously extracted DNA and RNA from manually micro-dissected SNpc biopsies at 14d p.i. (Scheme Fig. 1A). The DNA of two mice per condition, selected for high expression by RT-qPCR of Gadd45b or mCherry, respectively, was then subjected to reduced representation bisulfite sequencing (RRBS) for DNA methylation analysis. Bioinformatic analysis of RRBS data detected 809117 CpGs that were common between both conditions. Of those, 76185 individual CpGs were differentially methylated (DMCs) with a q-value smaller than, or equal to, 0.01 and at least $25 \%$ difference. Using a window and step size of $1000 \mathrm{bp}$, differentially methylated regions (DMRs) were defined and 16702 regions passed the defined 142 significance threshold. A majority of DMCs and DMRs was hypomethylated throughout time, located in open sea regions (defined as regions outside CpG

144 islands, CpG shores or CpG shelves) and localized in gene bodies, particularly introns. The Volcano plot in Figure 1D illustrates the DMC methylation pattern 14d p.i.. Upon Gadd45b overexpression, 46508 DMCs (61.05 \%) were hypomethylated and 29677 DMCs (38.95\%) were hypermethylated. The percentage of hypo- and 
hypermethylated regions per chromosome was similar (Suppl. Fig. 1G). We then interrogated DMCs in relation to their distance to a CpG island and found $69.73 \%$ to be overlapping with open sea regions (Fig. 1E), defined as regions outside of a known CpG island, CpG shore (2000 bp flanking the CpG island) or CpG shelves (2000 bp flanking the CpG shores). Analysis of the genomic context revealed that $41.36 \%$ of DMCs were located in introns (Fig. 1F), followed by $22.3 \%$ in the intergenic space, $23.6 \%$ in exons and $12.5 \%$ in promoter regions. This analysis indicates early and widespread methylation changes primarily in gene bodies upon Gadd45b overexpression.

In order to understand the long-term changes in methylation patterns induced by Gadd45b overexpression, we extracted DNA from mice 90 days after injection of AAV8-mGadd45b or AAV8-mCherry control. Analysis revealed a similar distribution of hypo- and hypermethylated DMRs at 14d p.i. (Suppl. Fig. 1A) and at 90d p.i. (Suppl. Fig. 1C). The genomic context of DMCs and DMRs, both at 14d p.i. (Suppl. Fig. 1B) and at 90d p.i. (Suppl. Fig. 1D) was very similar as well, the majority of differential methylation concerning open sea regions and gene bodies, particularly introns (summarized in Table 1). The overall numbers of DMRs and DMCs with AAV8-mGadd45b also resembled that at $14 \mathrm{~d}$ p.i., but common DMRs or DMCs examination revealed an overlap of only $15 \%$ of DMRs (2517) and $18 \%$ of DMCs (13920) (Fig. 1G). While this indicates that the specific regions with methylation changes induced by Gadd45b overexpression are not stable over time, the general location in open sea regions and in introns of genes of DMRs and DMCs is maintained as a specific and stable footprint of Gadd45b overexpression, which we termed "Gadd45b-regulon". Furthermore, there was an important overlap of more than half of the genes containing intronic hypomethylated or hypermethylated DMCs at $14 \mathrm{~d}$ and at $90 \mathrm{~d}$ (Fig. $\mathbf{1 H}$ ). The extent of overlap was similar for intronic DMRs (hypoDMRs at 14d: 3937 genes; hypoDMRs at 90d: 3467 genes, overlap (1712 common genes); Suppl. Fig. 1E). Of those common genes, 2353 genes contained at least one intronic hypo- and one intronic hypermethylated DMC (Fig. 1H) and 447 genes at least one intronic hypo- and one intronic hypermethylated DMR at both 14d and 90d p.i. (Suppl. Fig. 1E). Thus, Gadd45b overexpression induces stable changes in methylation patterns in gene bodies, particularly introns.

The results of the RRBS analysis prompted us to identify functional categories associated with this GADD45B-induced methylation footprint corresponding to the 
2353 genes stably containing DMCs over time (Fig. 1H). We used Gene ontology analysis (PANTHER version 15.0; http://pantherdb.org) and the PANTHER overrepresentation test with the GO-Slim annotation data set 'biological process'. 145 GO categories were significantly overrepresented with a fold change $>2$ and an FDR $<0.05$. The first fifteen significantly overrepresented GO categories are displayed in Figure 1I. Of those, $10 \mathrm{GO}$ categories are explicitly related to neuronal functions with two prevailing categories, namely synaptic function and organization, and neurodevelopment and neurogenesis. This is very similar to what we found for DMRs (Suppl. Fig. 1F) and suggests that Gadd45b is involved in the specific regulation of gene body methylation of neuron-related genes.

\section{Gadd45b overexpression leads to heterochromatin disorganization in mDA neurons}

Members of GADD45 protein family have been described to promote heterochromatin relaxation ${ }^{31}$. We therefore examined whether Gadd45b overexpression, in addition to global methylation changes, would also alter chromatin organization, in particular the organization of heterochromatin. To do so, we stained mDA neurons for histone $\mathrm{H} 3$ lysine 9 trimethylation (H3K9me3), a repressive heterochromatin mark. Immunostaining for H3K9me3 shows a perinucleolar pattern composed, on average, of 3 or 4 foci $(3.64 \pm 0.12)$ in $\mathrm{TH}+$ neurons in the SNpc of AAV8-mCherry injected mice (Fig. 2A). This pattern becomes disorganized in AAV8mGadd45b injected mice at $14 d$ p.i.. Semi-automated quantification of H3K9me3 staining specifically in $\mathrm{TH}+$ neurons identified a 1.13-fold increase in the number of H3K9me3 foci $(4.10 \pm 0.12)$ scattered across the nucleus, an increase by 1.24 -fold of the average $\mathrm{H} 3 \mathrm{~K} 9 \mathrm{me} 3$ foci volume $\left(4.78 \pm 0.15 ; 5.91 \pm 0.22 \mu \mathrm{m}^{3}\right)$, a reduction by 1.21 fold in the intensity of the diffuse nucleoplasmic H3K9me3 staining $\left(2.37 \times 10^{7} \pm 557961 ; 1.96 \times 10^{7} \pm 441292\right)$, but no difference in foci intensity $\left(1.32 \times 10^{6} \pm 54236 ; 1.41 \times 10^{6} \pm 64942\right)$ (Fig. 2A, quantification in Fig. 2B-E). This shift in heterochromatin organization is still detectable at $90 \mathrm{~d}$ p.i. (Fig. 2F-J). These results show that a de-structuration of heterochromatin is already detectable $14 \mathrm{~d}$ and stable up to $90 \mathrm{~d}$ after the injection of AAV8-mGadd45b indicating an early and stable perturbation of global heterochromatin organization upon Gadd45b overexpression.

We next examined whether Gadd45b overexpression leads to perturbations in the pattern of the DNA methylation marker MeCP2. Immunostaining of sections from mice injected with AAV8-mGadd45b or AAV8-mCherry did not show any difference in 
216 the number of MeCP2 foci in TH+ neurons (Suppl. Fig. 2A-D), the intensity of the 217 diffuse nucleoplasmic MeCP2 staining nor the volume and intensity of MeCP2 foci, neither at 14d nor at 90d p.i. (Suppl. Fig. 2E,G-J). There was a slight increase in foci intensity at 14d p.i. with AAV8-mGadd45b (Suppl. Fig. 2F). This analysis indicates that DNA methylation perturbation as detected by RRBS does not imply a global change in the organization of MeCP2 distribution.

Gadd45b overexpression leads to the loss of $\mathrm{TH}+$ neurons

To examine the effect of Gadd45b overexpression on the survival of mDA neurons in the SNpc, we quantified the number of $\mathrm{TH}+$ neurons in mice injected with AAV8-mCherry or AAV8-mGadd45b at $14 d$ and $90 d$ p.i. (Fig. 3A). There was no difference between $\mathrm{TH}+$ cell numbers comparing AAV8-mCherry- and AAV8$m$ Gadd45b-injected (ipsilateral) sides to the non-injected side (contralateral) at $14 \mathrm{~d}$ p.i. (Fig. 3B: AAV8-mCherry ipsi/contra: 1.04 \pm 0.02 ; AAV8-mGadd45b ipsi/contra: $0.97 \pm 0.04)$. However, $90 \mathrm{~d}$ after injection of AAV8-mGadd45b, this ratio shifted to an average of $0.82 \pm 0.04$, indicating a specific loss of $18 \%$ of $\mathrm{TH}+$ neurons on the AAV8mGadd45b injected side compared to the contralateral side (Fig. 3C). The TH+ neurons ratio between the ipsi- and the contralateral side remained unchanged in AAV8-mCherry injected mice (Fig. 3C: ipsi/contra: 0.97 \pm 0.02 ). These results show that Gadd45b overexpression in the SNpc can trigger degeneration of mDA neurons in the long-term. In addition, the absence of a significant $\mathrm{TH}+$ cell loss at $14 \mathrm{~d}$ p.i. suggests that alterations in the distribution of methylation at $\mathrm{CpGs}$ and in the organization of chromatin precede neuronal death.

Enhanced vulnerability of mDA neurons overexpressing Gadd45b to oxidative stress

To examine whether the heterochromatin de-structuration observed in $\mathrm{TH}+$ neurons at $14 d$ p.i. upon Gadd45b overexpression could render these neurons more vulnerable to oxidative stress, mice were first injected with AAV8-mCherry or AAV8mGadd45b and 14d later with 6-hydroxy-dopamine (6-OHDA, $2 \mu \mathrm{l} ; 0.5 \mu \mathrm{g} / \mu \mathrm{l}$ ) in the ipsilateral striatum. When injected into the striatum, 6-OHDA induces a specific and retrograde death of $\mathrm{mDA}$ neurons in the SNpc and is frequently used to model PD in rodents ${ }^{32}$ including mice ${ }^{33}$. Three days after the unilateral, striatal injection of 6-

248 OHDA, immunostainings of striatal sections show a $29 \%$ loss (mCherry ipsi/contra: $2490.71 \pm 0.04)$ of $\mathrm{TH}$ intensity in the ipsilateral striatum compared to the contralateral 
250

251

252

253

254

255

256

257

258

259

260

261

262

263

264

265

266

267

268

269

270

271

272

273

274

275

276

277

278

279

280

281

side in mCherry expressing mice (illustrated in Fig. 3D, quantified in Fig. 3E, left panel). This decrease in TH staining intensity reaches $44 \%$ (mGadd45b ipsi/contra: 0.56 \pm 0.04 ) in AAV8-mGadd45b injected mice (Fig. 3D, right panel), suggesting that Gadd45b overexpression increases the axonal degeneration of mDA neurons induced by 6-OHDA. However, this experimental paradigm did not lead to any significant loss of TH cell bodies in the SNpc (Fig. 3F), potentially due to the time of analysis. We analyzed mice 3 days rather than 6 days after 6-OHDA injection, the time point normally used to induce mDA cell death in the $\mathrm{SNpc}{ }^{32}$, to identify early events in $\mathrm{TH}+$ cell bodies. An increased vulnerability to oxidative stress of $\mathrm{mDA}$ neurons overexpressing Gadd45b was also reflected at the heterochromatin level. The injection of 6-OHDA changed the nuclear localization and increased the number of H3K9me3 positive foci (Fig. 3G, H) compared to AAV8-mCherry. Under these conditions, the number of MeCP2 positive foci in mDA neurons expressing AAV8mGadd45b was also increased as compared to AAV8-mCherry (Fig. 3l, J). This presumably reflects more significant DNA methylation changes upon Gadd45b overexpression under oxidative stress.

\section{Chromatin de-structuration is accompanied by increased DNA damage}

The heterochromatin loss model of aging stipulates that heterochromatin decondensation is a driving force of cellular aging ${ }^{34}$. Loss of proteins involved in heterochromatin maintenance has been shown to lead to increased DNA damage and accelerated aging ${ }^{35,36}$. In the context of NDs, recent studies have reported that chromatin relaxation in the brain could also result in increased DNA damage and genome instability ${ }^{18,37-39}$. Since Gadd45b overexpression led to chromatin changes, we investigated whether it might also induce DNA damage. We therefore performed immunostainings for phosphorylated histone $\operatorname{H} 2 A X(\gamma-H 2 A X)$, a marker for DNA strand breaks (Fig. 4A). TH+ neurons contained either a single perinucleolar focus or a diffuse nucleoplasmic staining without any foci. After injection of AAV8-mGadd45b (14d p.i.), the majority $(65.94 \pm 2.10 \%)$ of $\mathrm{TH}+$ neurons displayed a diffuse, intense nucleoplasmic staining against only $39.81 \pm 2.40 \%$ of $\mathrm{TH}+$ neurons after AAV8$m$ Cherry injection, the majority showing one or more prominent perinucleolar $\gamma$ $\mathrm{H} 2 \mathrm{AX}$-positive foci (Fig. 4B, C). The quantification of the diffuse $\gamma-\mathrm{H} 2 \mathrm{AX}$ staining in 
the nucleus revealed a more intense staining after Gadd45b overexpression, indicating widespread DNA damage.

\section{LINE-1 methylation is affected by Gadd45b overexpression}

Heterochromatin alterations can unsilence normally repressed TEs, including LINE-1 elements ${ }^{14}$. LINE-1 are a potential source of DNA damage ${ }^{16-18,40}$. Repeat elements are detected by RRBS and the Bismark software used for mapping of the RRBS reads only considers uniquely mapped reads to avoid any bias during the methylation calling. Thus, reads that are mapping with a same mapping score to multiple locations on the reference genome, which will be the case for most reads derived from repetitive elements, will not be considered. We exploited these facts to explore the methylation status of LINE-1 elements in our experimental conditions. Using the RRBS data, we used the LINE-1 annotation included in the software HOMER, which contains its own database of LINE-1 elements for the mouse genome to interrogate the overlap of DMRs and DMCs with LINE-1 elements 14 days after injection of AAV8 viruses. This analysis showed that 3530 DMCs and 1030 DMRs overlapped with an annotated LINE-1 element, which we termed L1-DMCs and L1DMRs respectively. 794 (22.5\%) L1-DMCs (Fig. 5A) and 264 (25.6\%) L1-DMRs (Suppl. Fig. 3A) were located in intronic regions, and 2734 (77.5\%) L1-DMCs (Fig. 5A) and 766 L1-DMRs (Suppl. Fig. 3A) in intergenic regions. Of all L1-DMCs the majority was hypomethylated (1933, 54,8\%, Fig. 5B), similar to L1-DMRs (595, $57,8 \%)$.

\section{To determine which LINE-1 families containing at least one L1-DMC upon} Gadd45b overexpression were overrepresented, we counted the number of families and ordered them by frequency. Figure $5 \mathrm{C}$ shows the ten most represented LINE-1 families with a L1-DMC. Interestingly, of 94 different families present, three LINE-1 family members, namely L1-MdF2 (975 L1-DMCs), L1Md-T (349 L1-DMCs) and L1Md-A (280 L1-DMCs), were the three most frequently represented. L1Md-T, L1MdA and L1-Md_F2 elements are young LINE-1 elements ${ }^{41}$, which contain full-length, retrotransposition-competent LINE-1 copies. Of LINE-1 associated intronic DMCs (L1-iDMCs, 794) at 14d p.i., the majority (54.7\%) was hypomethylated (434 hypomethylated vs 360 hypermethylated, Fig. 5D). L1-iDMCs were mostly located in protein-coding genes $(82.8 \%$, Fig. $5 E$ ) and the GO analysis of biological processes 
of 483 genes, containing at least one L1-iDMC, identified seven enriched categories. Among those categories, three were neuron-related (Fig. 5G). The most frequent LINE-1 families in intronic L1-iDMCs were, again, the young L1Md-F2 family (139 of 794) and L1Md-T (57 L1-iDMCs) followed by the old Lx8 family (57 L1-iDMCs) (Fig. 5F). Notably, other young LINE-1 families were also overlapping with iDMCs (L1MdA: 27 L1-iDMCs, L1Md-Gf: 6 L1-iDMCs, not shown) (Fig. 5F). Similarly to L1-iDMCs, the majority of LINE-1 associated DMRs in introns (L1-iDMRs) was hypomethylated (63,3 \%, 167 L1-iDMRs; Suppl. Fig. 3A) and belonged to the L1Md-F2 family (39 of 264) (Suppl. Fig. 3B). Hypo- and hypermethylated iDMRs were also found in LINE-1 elements of the active L1Md-T ( 3 and 2, respectively) and L1Md-A families (3 and 3 elements, respectively). This prompted to examine a data-base annotating full-length LINE-1 elements (L1Basev2 ${ }^{42}$ ) to see if intronic and intergenic DMRs and DMCs could coincide with possibly active LINE-1. Ten DMRs and 62 DMCs overlapped with a full-length LINE-1. More than half of them (7 out of 10 DMRs and 38 out of 62 DMCs, Fig. 5H, Suppl. Fig. 3C), were hypomethylated. This data indicates a widespread change in the methylation status of LINE-1 elements upon Gadd45b overexpression (14d p.i.). The most differentially methylated LINE-1 elements are hypomethylated and belong to young L1 families (L1Md-F2, L1Md-T, L1Md-A) suggesting a possible expression of these individual LINE-1 loci. Interestingly, L1iDMCs are frequently located in introns of genes related to neuronal functions.

LINE-1 transcripts are increased upon overexpression of Gadd45b

Having established a change in the methylation pattern of LINE-1 elements after injection of AAV8-mGadd45b, some of which were full-length, we evaluated the expression of the youngest L1 families in mice, namely L1Md-A and L1Md-Tf/Gf. The analysis by RT-qPCR with specific primers located in the 5'UTR of the L1Md-A (Fig. 5I, left panel) and L1Md-Gf/Tf families (Fig. 5l, right panel) showed a 1.5-fold increase in LINE-1 transcripts in the SNpc after 14d of Gadd45b overexpression.

Gadd45b overexpression is accompanied by expression changes in genes with DMCs

Expression levels of candidate genes with intronic DMCs at either $14 \mathrm{~d}$ or $90 \mathrm{~d}$ p.i. were then analyzed by RT-qPCR. We selected 13 genes based on their known function either in chromatin remodeling (Satb1, Setdb1, Wapl), DNA methylation 
350 (Tet2, Tet3, Dnmt3a), PD relevance (Lrrk2, Park2), synaptic remodeling (Sorcs2), 351 DNA damage (Xpa), or in aging and senescence (Cdkn2a-p19, Cdkn2d, Sirt1). None 352 of the candidate genes showed a change in expression at $14 d$ p.i. However, at $90 d$ 353 p.i., the expression of Satb1, Setdb1, Dnmt3a, Tet3 and Park2 decreased 354 significantly in the ipsilateral SNpc injected with AAV8-mGadd45b compared to 355 AAV8-mCherry. (Fig. 6A). Of note, 4 out of these 5 are genes belonging to the 356 Gadd45b-DMC-regulon (Fig. 1H). Using RNA-seq data of laser-microdissected SNpc 357 from wild-type mice (GEO GSE72321; 30 and unpublished), we compared the 358 expression levels of the selected candidate genes to those of dopaminergic neuron359 specific markers such as $T H$, dopamine transporter S/c6a3 or the homeogene, 360 Engrailed $1(E n 1)^{43}$. We found high levels of expression of Satb1 compared to the 361 other selected genes, indicating that Satb1 is strongly expressed in the SNpc (Suppl. 362 Fig. 4). Exonic and intronic DMCs over downregulated genes comprised hypo- as 363 well as hypermethylated CpGs. Disruption of the normal gene body methylation state 364 of several genes of the GADD45B-DMC-regulon might thus provoke their 365 dysregulation over time but is not associated with a loss of a particular methylation 366 pattern. 


\section{Discussion}

Epigenetic alterations and chromatin relaxation are hallmarks of aging ${ }^{2,3}$, but evidence of age- and disease-related global changes in the epigenetic landscape of different neuronal populations is still scarce. This information, however, holds important promises for understanding the implication of aging in the neuronal degeneration characterizing age-related NDs and might thus foster the understanding of the pathogenesis of NDs.

Here, we show that Gadd45b overexpression induces early changes in DNA methylation, particularly in introns of genes related to neuronal functions and on young and potentially active LINE-1 elements. This, accompanied by perturbations of heterochromatin organization and increased DNA damage culminate in neuronal cell death after several weeks. At an early time point before the onset of neurodegeneration, Gadd45b overexpression induces a vulnerable state in mDA neurons, increasing their sensitivity to oxidative stress induced by the striatal injection of 6-OHDA. This vulnerability is characterized by an amplified striatal dopaminergic axon terminal loss and an accentuation of perturbations in the organization of heterochromatin and DNA methylation in mDA cell bodies of the SNpc. We also demonstrate that LINE-1 transcripts are increased early on after Gadd45b overexpression. This increase in LINE-1, which are potent inducers of DNA damage in mDA neurons ${ }^{18}$, could explain the cell death of mDA neurons we observe when overexpressing Gadd45b long-term.

A number of recent studies indicate that GADD45 proteins play a key role in active DNA demethylation in post-mitotic neurons in the brain ${ }^{27,28}$ by serving as scaffolding proteins to recruit DNA repair enzymes such as the thymidine DNA glycosylase (TDG) to the site of DNA demethylation ${ }^{44}$. So far, GADD45B-regulated DNA demethylation has been described in the context of adult neurogenesis ${ }^{29}$, depressive-like behavior in mice ${ }^{45}$, major psychosis in humans ${ }^{46}$ and cerebral cortex plasticity ${ }^{47}$ on specific promoters, mostly on promoters of the Bdnf gene. Unexpectedly, when overexpressing Gadd45b in the SNpc of wild-type mice, we observed very limited changes in methylation on gene promoters, but rather widespread methylation changes on gene bodies with both, hypermethylated and hypomethylated CpGs. This apparent discrepancy with previous studies in terms of extent, localization and pattern of the DNA methylation changes induced by 
GADD45B might be due to methodology. Indeed, most studies in the brain did not use techniques allowing for unbiased global DNA methylation surveillance.

GADD45B has been shown to associate with TET (Ten-eleven translocation) proteins ${ }^{48,49}$ which transform 5 -methylcytosines $(5 \mathrm{mC})$ through a series of sequential oxidations. Those modified cytosines are excised by TDG and then replaced by nonmethylated cytosines through BER-dependent mechanisms ${ }^{50}$. A recent study has shown that Tet2 expression is increased in PD patients leading to altered $5 \mathrm{mC}$ patterns in enhancers of neuronal genes. Conversely, TET2 loss in mDA neurons was neuroprotective ${ }^{51}$. Tet2 did not show any changes in expression at either time point after AAV8-mGadd45b injection. This might be explained by the fact that Tet2 has many splice variants, which were not all covered in our RT-qPCR assay. However, Tet3 is down-regulated 90d after AAV8-mGadd45b injection. TET3 has been shown to interact with transcriptional regulators and histone writers such as H3K36 methyltransferases and to allow the active transcription of certain neuronal genes ${ }^{52}$. TET3 has also been reported to bind DNA and prevent aberrant methylation at the transcription start site of genes involved in lysosomal functions, mRNA processing and the BER pathway, pointing to a possible relevance of TET3 in the pathogenesis of NDs ${ }^{53}$. While our knowledge of how genome-wide DNA methylation patterns or epigenetic changes are correlated with PD pathogenesis is still scarce, there is evidence supporting that methylation changes in the SNpc correlate with aging ${ }^{23}$ and with cognitive impairment in PD ${ }^{54}$. Most of the existing data concerns methylation patterns of specific genes associated with NDs. Expression of the SNCA gene, encoding $\alpha$-synuclein and mutated in familial PD, for example, is under the control of DNA methylation ${ }^{55,56}$. Chromatin modifications on specific genes have also been reported, and the expression of several genes mutated in PD are modulated by histone modifications, including SNCA and MAPT encoding Tau ${ }^{25}$. Differentially methylated enhancers have been reported in $A D$ patients, and a dysregulation of histone acetylation in both, AD and PD patients ${ }^{57-60}$.

By serving as an adaptor between repair factors and chromatin, GADD45B can be seen as a communicating platform between DNA repair and epigenetics ${ }^{28,61}$. This could provide an explanation to the fact that we observe global changes in heterochromatin organization in addition to changes in DNA methylation. In line with this, GADD45 proteins have been reported to induce heterochromatin relaxation during cellular reprogramming ${ }^{31}$. GADD45B might thus be part of protein 
coordinators which link DNA methylation and histone modifications ${ }^{60}$. Perturbations of DNA methylation and global changes in heterochromatin organization, as we show here, induce neurodegeneration of mDA neurons in the SNpc with time and might thus be primary drivers of neurodegeneration.

Another primary hallmark of aging is genomic instability and some data suggest that this is also true in neuronal aging ${ }^{22}$. In this context it is important to note, that DNA strand breaks are physiologically occurring in post-mitotic neurons. This physiological process, however, needs to be tightly regulated, since the induction of DNA strand breaks is pathologically exacerbated in $A D{ }^{62}$. We have shown earlier, that DNA damage in $\mathrm{mDA}$ neurons can be induced by acute and chronic oxidative stress ${ }^{18,30}$ and that LINE-1 activity participates in DNA damage ${ }^{18}$. In the latter study, DNA damage was prevented either by siRNA against LINE-1 ORF2, the LINE-1 repressive protein Piwil1 or a nucleoside analogue reverse transcriptase inhibitor in vitro ${ }^{18}$. This LINE-1 induced DNA damage is dependent on young and active LINE-1 copies. These young LINE-1 elements belong mainly to the L1Md-Tf/Gf, A and $F$ families. In this context it is interesting to note that we observe the most pronounced changes in DNA methylation upon Gadd45b overexpression on these young LINE-1 families (Fig. 5). Intronic DMCs and DMRs, intronic L1associated DMCs (L1-iDMCs), and DMCs or DMRs overlapping with full-length LINE1 (flL1-iDMCs/flL1-iDMRs), are majorly hypomethylated and iDMCs are preferentially located in genes related to neuronal functions. It is therefore conceivable that the changes in methylation patterns we observe upon Gadd45b overexpression, particularly on young LINE-1 elements, are functionally linked to the increase in LINE-1 transcripts we observe 14 days after injection of AAV8-mGadd45b. Furthermore, based on previous evidence summarized above, this increase in expression of young $L 1$ elements of the $L 1 M d-T f / G f, A$ and $F$ families might be at the origin of the DNA damage in mDA neurons upon Gadd45b overexpression.

Several lines of evidence suggest that the activation of TEs might be associated with aging and NDs. The expression of LINE-1 in wild-type mice increases in neurons ${ }^{63}$, liver and muscle during aging ${ }^{64}$. The activation of TEs with aging leads to neuronal decline and shorter lifespan in drosophila ${ }^{65,66}$ and increased TE expression has been reported in brain tissue from PD, AD and ALS patients ${ }^{37,67}$. Recently, elevated transcripts of repetitive sequences have been found in the blood of PD patients ${ }^{68}$. Heterochromatin de-structuration and increased TE activity lead to 
469

470

471

472

473

474

475

476

477

478

479

480

481

482

483

484

485

486

487

488

489

490

491

492

493

494

495

496

497

498

499

500

501

502

an AD-like phenotype in a mouse model with targeted disruption of $B m i 1$, a gene involved in heterochromatin maintenance and altered expression in AD patients ${ }^{39}$. In $A D$ and in ALS, the Tau protein as well as TDP-43 can induce heterochromatin relaxation, especially at the level of LINE-1 elements in the case of TDP-43, leading to increased TE activity and neurotoxicity ${ }^{37,67,69}$. An aging-induced activation of TEs, which are intrinsic components of the genomes of virtually all eukaryotes, might link genomic instability and epigenetic changes to the aging process.

Changes in chromatin states lead to changes in gene expression as exemplified during the transition from neuronal progenitors to adult neurons ${ }^{70}$ and during aging ${ }^{71}$, and recent data suggests that this could also be the case in NDs 59,72. In several NDs (AD and HD ${ }^{73,74}$; ALS ${ }^{75}$ ), disease-specific gene expression changes are increasingly recognized, but whether they overlap with expression profiles characteristic to aging is not known yet. In AD patients, widespread loss of heterochromatin was accompanied by a transcriptomic profile resembling the one of a fetal brain ${ }^{38}$, suggesting that the relaxation of chromatin allows the expression of normally repressed genes which alters several biological processes and leads to neurodegeneration neurodegeneration ${ }^{76}$. This is also in line with our data showing the preferential location of iDMCs and iDMRs in genes involved in neurogenesis but it remains to be seen whether their expression is altered. Interestingly, it has been shown that Gadd45b activity promotes adult neurogenesis ${ }^{29}$. The majority of these genes with iDMCs and iDMRs upon overexpression of Gadd45b belonged to neuronal categories, particularly synapse-related and neurodevelopmental categories. In this context it is interesting to note that synaptic homeostasis is an emerging key player in the pathogenesis of $P D{ }^{77}$ and synaptic dysfunction is an early event in neurodegeneration ${ }^{78}$. Gadd45b overexpression induced DMCs and DMRs preferentially in introns of genes. The role of gene body or intronic methylation on gene expression is not completely understood ${ }^{79}$. In recent years, several studies have reported that gene body methylation influences gene expression levels and/or alternative splicing 808182 . Among the list of genes underlying a direct or indirect regulation through methylation changes triggered by Gadd45b overexpression, the decrease in expression did not correlate with a specific direction of methylation changes towards hypo- or hypermethylated $\mathrm{CpGs}$ but rather with a change in the methylation state throughout the gene body. Satb1 has been described as a dopaminergic-specific regulator of senescence ${ }^{83}$, a dopaminergic neuron cell 
503 survival factor ${ }^{84}$ and a regulator of global chromatin structuration ${ }^{85,86}$. The decline in

504 Satb1 expression at $90 \mathrm{~d}$ p.i. might help to explain, at least partly, the

505

506

507

508

509

510

511

512

513

514

515

516

517

518

519

520

521

522

523

524

525

526

527

528

529

530

531

532

533

534

535 neurodegeneration of mDA neurons upon Gadd45b overexpression. Setdb1 is a histone-lysine-methyltransferase that specifically trimethylates lysine-9 of histone $\mathrm{H} 3$. It is tempting to speculate that the decrease in expression might relate to the change in the organization of H3K9me3 we observe upon Gadd45b overexpression. Dnmt3a, a genome-wide de novo DNA methyltrasferase, and Tet3, are involved in DNA methylation or demethylation, respectively. Interestingly, inactivation of TET and/or DNMT proteins causes gains and losses of DNA methylation, suggesting that the loss of one regulator can lead to the redistribution of other regulators and of DNA modifications ${ }^{87}$. Park2, encoding the ubiquitin protein ligase Parkin, is a PD-related gene and loss-of-function mutations in this gene are responsible for familial forms of PD. It is thus possible, that a decrease in the expression of Park2 in the context of Gadd45b overexpression might contribute to the degeneration of mDA neurons. Overall, the expression changes might be due to the Gadd45b-induced epigenetic dysregulation of the neuronal genome and participate in the Gadd45b overexpression phenotype and the challenge will be to correlate one with the other.

Altogether, our data are in line with an emerging concept on a new pathogenic pathway initiating age-related neurodegeneration. Recent evidence, including from our group, suggests that aging-induced chromatin reorganization triggers the activation of LINE-1 retrotransposons and subsequent LINE-1 induced DNA damage cumulating in neuronal cell death. Our group has shown that acute and chronic oxidative stress leads to heterochromatin relaxation and LINE-1 activation in mDA neurons in vivo ${ }^{18,30}$. Aging-induced epigenetic alterations might produce a vulnerable pre-ND state. Combining this pre-ND state with a particular genetic susceptibility, a familial gene mutation or an accelerating environmental trigger, could initiate a cascade of secondary events including protein aggregation, metabolic dysregulation and mitochondrial dysfunctions. NDs share several common pathological features and despite extensive investigation, no disease-modifying treatment is available. Acknowledging aging as a vulnerability factor for neurodegeneration is important not only for understanding the pathogenesis of NDs, but also for modeling, testing and developing therapeutics for crucially lacking disease-modifying treatments. Our study suggests two novel therapeutic targets for neuroprotection. Drugs restoring 
bioRxiv preprint doi: https://doi.org/10.1101/2020.06.23.158014; this version posted June 23, 2020. The copyright holder for this preprint (which was not certified by peer review) is the author/funder, who has granted bioRxiv a license to display the preprint in perpetuity. It is made available under aCC-BY-NC-ND 4.0 International license.

536 chromatin structure and/or repressing LINE-1 transcription or activity might hold 537 promise for the prevention of age-related neurodegeneration. 


\section{Materials and Methods}

539

540

541

542

543

544

545

546

547

548

549

550

551

552

553

554

555

556

557

558

559

560

561

562

563

564

565

566

567

568

569

570

\section{Animals}

All animal treatments followed the guidelines for the care and use of laboratory animals (US National Institutes of Health), the European Directive number 2010/68/UE (EEC Council for Animal Protection in Experimental Research and Other Scientific Utilization). This project was validated by the competent ethical committee (CEA 59) and authorized by the Minister of Higher Education, Research and Innovation ( $\mathrm{n}^{\circ} 00703.01$ and $\mathrm{n}^{\circ}$ APAFIS\#6605-2016090209434306 v3). For surgical procedures, animals were anesthetized with Xylazine (Rompun 2\%, $5 \mathrm{mg} / \mathrm{kg}$ ) and Ketamine (Imalgene 1000, $80 \mathrm{mg} / \mathrm{kg}$ ) by intraperitoneal injection and a local subcutaneous injection of lidocaine $(0.5 \%, 3 \mathrm{mg} / \mathrm{kg})$ on the incision site. Postchirurgical analgesia was insured by an injection of the analgesic Meloxicam (Metacam, 0,5mg/kg) s.c.. Swiss OF1 wild-type mice (Janvier) were maintained under a $12 \mathrm{~h}$ day/night cycle with ad libitum access to food and water. A maximum of 5 mice were housed in one cage, and cotton material was provided for mice to build a nest. Experimental groups consisted of five to seven male mice of 6 weeks of age. Sample size calculations were based on previous experiments.

\section{AAV8 vectors to overexpress Gadd45b}

Forced expression of Gadd45b in mDA neurons was achieved using an AAV8 viral vector. The constructs contained cDNAs for either mouse Gadd45b (AAV8mGadd45b) or mCherry (AAV8-mCherry) under the control of the ubiquitous EF1a promotor. Gadd45b cDNA was flanked by the cognate 5' and 3' UTRs (Fig. 1A). AAV8 was chosen because it has previously been shown to efficiently infect mDA neurons in the midbrain.

\section{Brain injections}

For injections, mice were placed in a stereotaxic instrument, and a burr hole was drilled into the skull $3.3 \mathrm{~mm}$ caudal and $1.3 \mathrm{~mm}$ lateral to the Bregma. The needle was lowered $3.8 \mathrm{~mm}$ from the surface of the skull, and AAV8-Ef1a-mCherry or AAV8Ef1a-mGadd45b (Vector Biolabs; $2 \mu \mathrm{l} ; 4.8 \times 10^{13} \mathrm{GC} / \mathrm{ml}$ suspended in $\mathrm{NaCl} 0,9 \%$ with $5 \%$ glycerol) injections were performed over $4 \mathrm{~min}$. Where indicated 6-OHDA (2 $\mu \mathrm{l}$; 
$5710.5 \mu \mathrm{g} / \mu \mathrm{l}$; Sigma) injections were performed in the same manner $0.4 \mathrm{~mm}$ rostral, 1.8

$572 \mathrm{~mm}$ lateral and $3.8 \mathrm{~mm}$ ventral to the bregma, over $4 \mathrm{~min}$.

573

\section{Tissue dissection}

575 For RNA and DNA analyses, biopsies of the SNpc were performed. Brains were put 576 into a custom-made brain slicer for adult mice brain. A coronal slice of $\approx 2 \mathrm{~mm}$ encompassing the SNpc was excised (Bregma $-3.26 \mathrm{~mm}$ to $-5.2 \mathrm{~mm}$ ) and placed on a cold cover slide with the rostral side facing the experimenter. Dissection of the SNpc was then done following anatomical landmarks: a sagittal cut to separate the two hemisphere, a second parasagittal cut through the fasciculus retroflexus and the mammillothalamic tract (about 2/3 starting from the midline of the distance between

582 the midline and the rostral end of cerebral peduncle) to remove the VTA, a

583 transversal section from the ventral part of the lateral geniculate complex to the 584 midline, a second transversal cut from the ventral end of the cerebral peduncle to the midline. The cerebral cortex was then removed to only keep the midbrain part containing the SNpc and immediately frozen on dry ice and kept at $-80^{\circ} \mathrm{C}$ until 587 extraction.

588

589

590

591

592

593

594

595

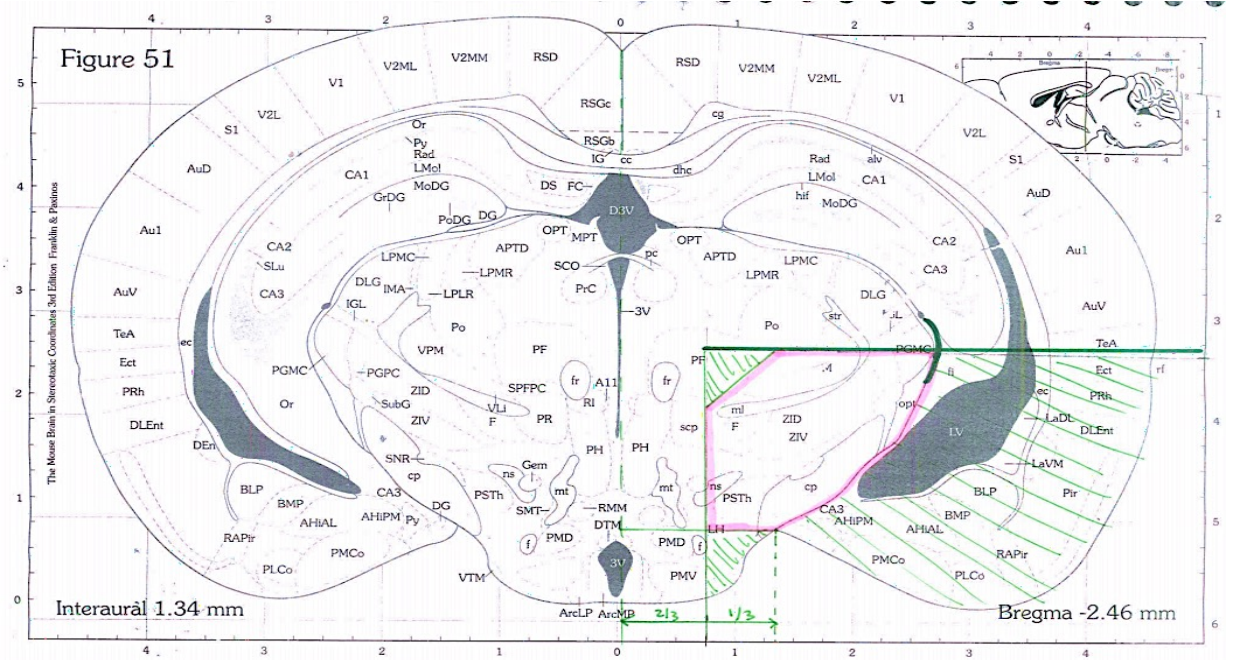

\section{$R T-q P C R$}

Total RNA from dissected SNpc was extracted using the AllPrep DNA/RNA Micro Kit (Qiagen 80284) adding an on-column DNase I treatment (Qiagen 79256), followed by RT-qPCR. RNA (200 ng) was reverse-transcribed using the QuantiTect Reverse Transcription kit (Qiagen 205313). Quantitative PCR reactions were carried out in duplicates with SYBR Green I Master Mix (Roche S-7563) on a LightCycler 480 
596 system (Roche Applied Science). The following primers were used: Hprt (sense:

597 AGCAGGTGTTCTAGTCCTGTGG, antisense: ACGCAGCAACTGACATTTCTAA);

LINE-1

A (sense:

TTCTGCCAGGAGTCTGGTTC,

antisense:

599 TGAGCAGACCTGGAGGGTAG);

LINE-1

$\mathrm{Tf} / \mathrm{Gf}$

(sense:

600

CTGGGAACTGCCAAAGCAAC,

antisense:

CCTCCGTTTACCTTTCGCCA);

601

Gadd45b

(sense:

ACTGATGAATGTGGACCCCG,

antisense:

602 CCTCTGCATGCCTGATACCC); Satb1 (sense: TCTTTTACCCCCTCCTCCCA,

603 antisense:

TCACCTGCCAGAACACTTCA);

Tet3

(sense:

604

605

CTCGGCGGGGATAATGGGAG,

antisense:

AGCCTGTCTTGACAGTCGCC);

606 Dnmt3a (sense:

GCCGAATTGTGTCTTGGTGGATGACA,

antisense:

CCTGGTGGAATGCACTGCAGAAGGA),

Setdb1

(sense:

607

608 (sense:

GCTCAAGGAAGTGGTTGCTAAG,

antisense:

CAATACTCTGTTGTTCCAGGTCA). Primer efficiencies were tested using 10-fold dilution series of cDNA spanning at least three orders of magnitude. Data were analyzed using the ddCt method and values normalized to hypoxanthine-guanine

DNA extraction and quantification phosphoribosyl transferase (Hprt).

DNA was also extracted during the same process of RNA extraction using the AllPrep DNA/RNA Micro Kit (Qiagen 80284). The DNA was then purified, treated with RNase H (ThermoFischer, 18021071) and Proteinase K (PCR grade, Roche, 3115836001) and concentrated with the DNA Clean \& Concentrator-5 kit (Zymo, D4013). DNA concentration of each sample was measured using the Qubit $\circledast$

\section{RRBS}

623 RRBS was performed by Diagenode. DNA quality of the samples was assessed with

624 the Fragment AnalyzerTM and the DNF-488 High Sensitivity genomic DNA Analysis Kit (Agilent). DNA was slightly more fragmented than defined by the quality control standards but this fragmentation was minor. RRBS libraries were prepared using the

627 Premium Reduced Representation Bisulfite Sequencing (RRBS) Kit (Diagenode) which uses the Mspl restriction enzyme and size selection to enrich for CpG-rich regions (coverage of about 4 million CpGs). 100ng of genomic DNA were used to 
630

631

632

633

634

635

636

637

638

639

640

641

642

643

644

645

646

647

648

649

650

651

652

653

654

655

656

657

658

659

660

661

662

663

start library preparation for each sample. Following library preparation, samples were pooled together by 8. PCR clean-up after the final library amplification was performed using a 1.45x beads:sample ratio of Agencourt $\circledast$ AMPure $®$ XP (Beckman Coulter). DNA concentration of the pools was measured using the Qubit ${ }^{\circ}$ dsDNA HS Assay Kit (Thermo Fisher Scientific). The profile of the pools was checked using the High Sensitivity DNA chip for 2100 Bioanalyzer (Agilent). RRBS library pools were sequenced on a HiSeq3000 (Illumina) using 50 bp single-read sequencing (SR50). Bisulfite conversion and amplification were performed using Diagenode's Premium RRBS Kit. After conversion, the pooled samples were analyzed by qPCR. Sequencing was performed in single-end mode, generating 50 bases reads (SE50) on an Illumina HiSeq 3000/4000. Quality control of sequencing reads was performed using FastQC version

0.11 .8 (https://www.bioinformatics.babraham.ac.uk/projects/fastqc/). Adapter removal was performed using

Trim Galore (https://www.bioinformatics.babraham.ac.uk/projects/trim_galore/) version $\quad 0.4 .1$. Reads were then aligned to the murine reference genome mm10/GRCm38 using bismark v0.20.0 ${ }^{88}$. Bismark is a specialized tool for mapping bisulfite-treated reads such as the ones generated in RRBS-seq experiments. Bismark requires that the referenced genome first undergoes an in-silico bisulfite conversion while transforming the genome into forward $(C \rightarrow T)$ and reverse strand $(G \rightarrow A)$ versions. The reads producing a unique best hit to one of the bisulfite genomes were then compared to the unconverted genome to identify cytosine contexts (CpG, $\mathrm{CHG}$ or $\mathrm{CHH}$ - where $\mathrm{H}$ is $\mathrm{A}, \mathrm{C}$ or T). The cytosine2coverage and bismark_methylation_extractor modules of Bismark were used to infer the methylation state of all cytosines (for every single uniquely mappable read) and their context, and to compute the percentage methylation. The reported cytosines were filtered to get only the $\mathrm{CpGs}$ covered in each sample. The spike-in control sequences were used at this step to check the bisulfite conversion rates and to validate the efficiency of the bisulfite treatment. Methylkit v1.7.0 ${ }^{89}$, a R/Bioconductor package, was used to perform the differential methylation analysis between the two groups of samples. The $\mathrm{CpG}$ data set was filtered for low coverage (CpGs with coverage less than 10X in all samples per comparative group were discarded) and for extremely high coverage to discard reads with possible PCR bias (CpGs with coverage higher than the 99.9th percentile were discarded). The data was then normalized for read coverage distribution between 
664

665

666

667

668

669

670

671

672

673

674

675

676

677

678

679

680

681

682

683

684

685

686

687

688

689

690

691

samples. A pairwise comparison was performed for first versus second group of samples to identify differentially methylated CpGs (DMCs) and differentially methylated regions (DMRs), the latter with a window and step size of 1000bp. Methylkit uses logistic regression to compare the methylation percentages between groups at a given $\mathrm{CpG} /$ region. All DMCs and DMRs were annotated with the $\mathrm{R} /$ Bioconductor package annotation ${ }^{90}$, with the refGene and $\mathrm{CpG}$ island annotations from UCSC. The annotation comprised two categories: (i) distance to a CpG island and (ii) regional annotation. The distance related annotation classified DMCs and DMRs whether they overlapped a known CpG island, $2000 \mathrm{bp}$ of the flanking regions of the CpG islands (shores), 2000 bp of the flanking regions of the shores (shelves) or outside these regions (open sea). The regional analysis classified DMCs and DMRs in four groups, namely, exons, introns, promoters and intergenic regions.

\begin{tabular}{|c|c|c|c|c|c|c|c|c|}
\hline $\begin{array}{c}\text { Sample } \\
\text { Name }\end{array}$ & Total reads & $\begin{array}{c}\text { Uniquely } \\
\text { aligned }\end{array}$ & $\begin{array}{c}\text { Mapping } \\
\text { efficiency (\%) }\end{array}$ & $\begin{array}{c}\text { CpGs } \\
\text { detected }\end{array}$ & $\begin{array}{c}\text { CpGs } \\
\text { cov>10 }\end{array}$ & $\begin{array}{c}\text { Average } \\
\text { Coverage }\end{array}$ & $\begin{array}{c}\text { Conv. Rate } \\
\text { Meth. } \\
\text { spike-in (\%) }\end{array}$ & $\begin{array}{c}\text { Conv. Rate } \\
\text { Unmeth. } \\
\text { spike-in (\%) }\end{array}$ \\
\hline mC1_2w & 32.674 .187 & 21.455 .193 & 65.66 & 1.552 .817 & 1.316 .296 & 36.96 & 1.76 & 99.34 \\
\hline mC4_2w & 45.583 .765 & 29.506 .694 & 64.73 & 1.786 .123 & 1.544 .593 & 38.73 & 1.56 & 99.53 \\
\hline mG1_2w & 39.606 .244 & 26.791 .258 & 67.64 & 1.727 .650 & 1.495 .577 & 40.53 & 1.48 & 99.30 \\
\hline mG6_2w & 43.911 .896 & 29.422 .323 & 67.00 & 1.742 .579 & 1.527 .329 & 43.18 & 1.56 & 99.47 \\
\hline mC1_3m & 43.426 .559 & 28.584 .928 & 65.82 & 1.719 .287 & 1.499 .415 & 40.19 & 1.31 & 99.51 \\
\hline mC3_3m & 37.983 .254 & 24.904 .215 & 65.57 & 1.644 .193 & 1.412 .632 & 37.57 & 1.64 & 99.42 \\
\hline mG1_3m & 41.810 .446 & 27.906 .101 & 66.74 & 1.665 .705 & 1.481 .471 & 44.30 & 1.73 & 99.52 \\
\hline mG4_3m & 37.699 .685 & 24.957 .045 & 66.20 & 1.652 .248 & 1.422 .694 & 36.72 & 1.72 & 99.47 \\
\hline
\end{tabular}

\section{Immunostaining}

For immunostaining, animals received a lethal intraperitoneal injection of $1 \mu \mathrm{l} / \mathrm{g}$ body weight dose of Euthasol (150mg/kg) and were then perfused with $8 \mathrm{~mL}$ of Phosphate Buffer Saline (PBS) then $8 \mathrm{~mL}$ of $4 \%$ Paraformaldehyde (PFA) at a rate of $300 \mathrm{ml} / \mathrm{h}$ using a syringe pump. Brains were then post-fixated 1 hour at room temperature (RT) in 4\% PFA, washed in PBS three times for 30 minutes at RT and placed in PBS with $20 \%$ sucrose overnight at $4^{\circ} \mathrm{C}$. After cryoprotection, the brains were embedded in Tissue Freezing Medium (TFM, Microm Microtech), frozen on dry ice and $30 \mu \mathrm{m}$ sections of mouse striatum and ventral midbrains encompassing the SNpc were prepared using an HM 560 Microm cryostat (Thermo Scientific).

Slides with $30 \mu \mathrm{m}$ striatum or midbrain sections were washed in PBS and permeabilized with $1 \%$ Triton X-100. After 30 minutes at RT in $100 \mu \mathrm{M}$ glycine buffer (for $\mathrm{TH} / \mathrm{mCherry}$ and $\mathrm{TH} / \mathrm{ORF} 1 \mathrm{p}$ ) or 30 minutes at $100^{\circ} \mathrm{C}$ in demasking citrate buffer (10 mM, pH 6, 0.05\% Tween) (for TH/MeCP2, H3K9 or $\gamma-\mathrm{H} 2 \mathrm{AX}$ ), sections were first 
692

693

694

695

696

697

698

699

700

701

702

703

704

705

706

707

708

709

710

711

712

713

714

715

716

717

718

719

720

721

722

723

724

725

blocked in 10\% Fetal Bovine Serum (FBS, Gibco) in the presence of 0.5\% Triton X100 for 1 hour at RT and incubated with primary antibodies overnight at $4^{\circ} \mathrm{C}$, washed and further incubated with secondary antibodies for 1 hour at RT. The following primary antibodies used: anti- $\gamma-\mathrm{H} 2 \mathrm{AX}$ (mouse, 1/200, Millipore, clone JBW301), antiTH (chicken, 1/500, Abcam, ab76442), anti-ORF1p (guinea pig, 1/200, in-house, clone 09 as in ${ }^{83}$, anti-mCherry (mouse, 1/200, Clontech 632543), rabbit antiH3K9me3 (rabbit, 1/200, Abcam, ab8898) and anti-MeCP2 (rabbit, 1/200, Millipore MABE328). Sections were incubated with appropriate secondary antibodies (488 anti-chicken, 546 anti-mouse, 647 anti-guinea pig, 647 anti-rabbit, 647 anti-mouse, Alexa Fluor, Life Technologies)

\section{In situ hybridization}

Mice were perfused with PBS in RNase-free conditions, and frozen in isopentane (embedded in TissueTek O.C.T). Brain slices $(20 \mu \mathrm{m})$ were fixed in $4 \%$ PFA in PBS for $10 \mathrm{~min}$ at RT and then permeabilized twice for $10 \mathrm{~min}$ in RIPA buffer (150 mM $\mathrm{NaCl}, 1 \% \mathrm{NP}-40,0.5 \% \mathrm{Na}$ deoxycholate, 0.1\% SDS, $1 \mathrm{mM}$ EDTA, $50 \mathrm{mM}$ Tris- $\mathrm{HCl}$ $\mathrm{pH} 8$ ). Brain sections were fixed again for $5 \mathrm{~min}$ in 4\% PFA, acetylated for $10 \mathrm{~min}$ in $0.25 \%$ acetic anhydride (in $0.1 \mathrm{M}$ triethanolamine, $\mathrm{pH} 8$ ). Sections were then permeabilized for 30 min in PBS with 1\% Triton X-100, 10 min in 10mM citrate buffer $\mathrm{pH} 6$ at a $100^{\circ} \mathrm{C}$ and then pre-incubated for $1 \mathrm{~h}$ at $70^{\circ} \mathrm{C}$ in hybridization buffer $(50 \%$ formamide, $5 \times$ SSC, $5 \times$ Denhardt (1\% Ficoll, 1\% SSC, 1\% Tween-20), $500 \mu \mathrm{g} / \mathrm{ml}$ Salmon sperm DNA, $250 \mu \mathrm{g} / \mathrm{ml}$ yeast tRNA). Sections were then hybridized overnight at $70{ }^{\circ} \mathrm{C}$ with a digoxigenin (DIG)-labeled RNA probes (DIG RNA labeling kit, Roche 11277073910) for Gadd45b mRNA. Sections were washed with FAM/SSC (50\% formamide, $2 \times$ SSC, $0.1 \%$ Tween-20) twice $30 \mathrm{~min}$ at $37^{\circ} \mathrm{C}$, then twice in $0.2 \times$ SCC at $42^{\circ} \mathrm{C}$, blocked in $\mathrm{B} 1$ buffer $(100 \mathrm{mM}$ maleic acid $\mathrm{pH} 7.5,150 \mathrm{mM} \mathrm{NaCl})$ with $10 \%$ fetal bovine serum (FBS) for $1 \mathrm{~h}$, and incubated overnight at $4^{\circ} \mathrm{C}$ in $\mathrm{B} 1$ buffer with alkaline phosphatase-conjugated anti-DIG (1/2000; Roche 11633716001). After three washes in B1 buffer and one wash in B3 buffer (100 mM Tris- $\mathrm{HCl}$ pH 9, 50 mM $\mathrm{MgCl}_{2}, 100 \mathrm{mM} \mathrm{NaCl}, 0.1 \%$ Tween-20) for $15 \mathrm{~min}$, slides were stained using the NBT/ BCIP kit (Vector labs, SK5400), stopped with $\mathrm{ddH}_{2} \mathrm{O}$ and slides were mounted with DAKO-mounting medium.

\section{Imaging/ Microscopy}


All large field images used for $\mathrm{TH}+$ neuron quantification, level of viral infection quantification and striatal intensity quantification were made on widefield microscope (Axio zoom V16 - Zeiss - Apotome.2) at 2,3 magnification with a zoom factor of 100. ISH image was taken by upright widefield microscope equipped with a color CCD camera (Nikon 90i microscope) at 20x magnification in brightfield. H3K9me3, MeCP2 and $\gamma-\mathrm{H} 2 \mathrm{AX}$ foci quantification as well as ORF1p intensity quantification were made on images taken by spinning disk microscopy (Yokogawa W1 Spinnning-disk head mounted on an inverted Zeiss AxioObserver Z1) at 63x magnification. confocal microscopy (LSM 980 with Airyscan 2, Zeiss) at 63x magnification with a zoom factor of 1.4, except for the MeCP2 and ORF1p images (performed on the spinning-disk microscope).

Cell counting and image quantification

740

741

742

TH cell counting in conditions comparing ipsi- (treated) and contralateral (nontreated) sides were done as follows: For every brain, a minimum of four serial sections were stained, and the number of TH cells was counted in the SNpc of both ipsi- and contralateral sides. An ipsi/contra ratio was calculated for each section, and the resulting mean of four sections was used to quantify the difference between the TH cell number of the ipsi- and contralateral side of the same animal. The counting was done blindly.

The quantification of axonal degeneration in the striatum comparing ipsi- and contralateral sides was done as follows: For every brain, a minimum of seven serial sections were stained, and the integrated density of $\mathrm{TH}$ staining intensity was measured in ImageJ by determining the entire contralateral striatum as region of interest (ROI) and conserving area for the measurement of ipsilateral sides. An ipsi/contra ratio was calculated for each section, and the mean ratio of sections containing the striatum was used to quantify the difference between $\mathrm{TH}$ striatal intensity of the ipsi- and contralateral side of the same animal. The quantification was done blindly.

Quantifications of foci were performed using a $63 \times$ magnification and $0.3 \mu \mathrm{m}$ thick successive focal planes except for $\gamma-\mathrm{H} 2 \mathrm{AX}$ foci quantification, which was made using $0.2 \mu \mathrm{m}$-thick successive focal planes. Immunostainings of one parameter (H3K9, MeCP2, Etc.) were all done in one experiment for all the conditions. For each 
760

761

762

763

764

765

766

767

768

769

770

771

772

773

774

775

776

777

778

779

780

781

782

783

784

785

786

787

788

789

790

791

792

immunostaining, 3 images of the SNpc per side were taken on 3 sections per mouse, thus 18 images per mouse ( $n=3$ per condition), or 54 images per condition. The same parameters were set-up on the spinning disk microscope to allow for comparison between experimental conditions for the same staining. Images were analyzed by the same experimenter using ImageJ software ${ }^{84}$. For foci quantifications the foci counting Fiji Plug-in was used ${ }^{85}$. In addition, an image analysis plug-in was developed for the ImageJ/Fiji software, using Bio-Format (openmicroscopy.org), mcib3D ${ }^{86}$ and GDSC (Alex Herbert from Sussex University) libraries. First, nuclei that belonged to $\mathrm{TH}+$ neurons were manually marked with the plug-in Cell Counter, an $\mathrm{xml}$ file for each image containing 3D nuclei coordinates was saved. Then, nucleus channel was filtered with a median filter (radius $=4$ ) and a Difference of Gaussian $(\mathrm{DOG})$ (sigma1 $=30$, sigma2 $=15$ ), a binary mask was done with an Otsu threshold. Only nuclei that are associated to the nuclei defined in the $\mathrm{xml}$ file were kept. MeCP2 and H3K9me3 foci detections were performed using a median filter (radius=2), DOG (sigma1=10, sigma2=2), binary mask was done with a MaxEntropy threshold, then 3D objects (foci) that had a volume comprised between 1.5 and 40 $\mu \mathrm{m}^{3}$ and were inside $\mathrm{TH}+$ nuclei or at a $2 \mu \mathrm{m}$ distance to nucleus was associated to nuclei. $\gamma$-H2AX foci detection was performed using a median filter (radius=2), DOG (sigma1=7, sigma2=3), binary mask was done with a Moments threshold, then 3D objects (foci) that had a volume comprised between 0.5 and $100 \mu \mathrm{m}^{3}$ were inside $\mathrm{TH}+$ nuclei or at a $2 \mu \mathrm{m}$ distance to nucleus was associated to nuclei. For each nucleus, foci number, average foci volume, average foci integrated intensity and average nucleus integrated intensity was computed.

\section{Gene ontology analysis}

Gene ontology analysis (PANTHER version $15.0 ;{ }^{87}$ ) was done using the PANTHER overrepresentation test with the GO-Slim annotation data sets 'biological process', 'molecular function' and cellular component' and the 'mus musculus' gene set as the reference list. Fisher's exact test was used to compute statistical significance of overrepresentation with the false discovery rate (FDR) set at $p<0.05$. The first 15 categories, ordered by fold enrichment, are displayed along with the corresponding FDR value. 
793

794

795

796

797

798

799

800

801

802

803

804

805

806

807

808

809

810

811

812

813

814

815

816

817

818

819

820

821

822

823

824

\section{Statistics}

Unless otherwise stated, the graphs represent each replicate and the error bar the SEM of the mean of replicates. Error bars, values of $n$ and mean \pm SEM are as stated in the figure legends. Results were considered as statistically significant for Pvalue $<0.05$; in some cases, the exact $P$-value is given. Normality test were performed prior to the statistical test and unless stated otherwise, the nonparametric Wilcoxon-Mann-Whitney test was applied. All statistical analysis was done with the software Prism.

For the bioinformatic analysis of the RRBS, we formulated the null hypothesis that there are no differences in methylation between the two groups. After the pvalues have been computed, Methylkit, an R package for DNA methylation analysis and annotation, uses the sliding window model (SLIM) to correct P-values to q-values for multiple comparison tests. Statistically significant DMCs and DMRs were identified with a q-value cutoff $<0.01$ and a methylation difference higher than $25 \%$.

\section{Acknowledgements}

This work was supported by the Fondation de France (00086320 to J.F.) and the Fondation du Collège de France (to J.F.). We thank all primary donors for their financial contributions to this work. We thank the animal facility members for their essential contributions. We gratefully acknowledge Julien Dumont and the Collège de France Orion imaging facility (IMACHEM-IBiSA), member of the French National Research Infrastructure France-Biolmaging (ANR-10-INBS-04), which received support from the program «Investissements d'Avenir» ANR-10-LABX-54 MEMOLIFE. We thank Yves Dupraz for the manufacturing of a customized mouse brain slicer.

\section{Author contributions}

CRG performed most of the experiments and participated in the writing of the manuscript, $O M B$ contributed experimentally, $P M$ designed the semi-automated image analysis workflow, AP co-supervised the beginning of the study, RLJ cosupervised the study and contributed to the writing of the manuscript, JF cosupervised the study, analyzed the RRBS data, wrote the manuscript and received the funding. 


\section{Figure Legends}

Figure1. Gadd45b overexpression in the SNpc of wildtype mice leads to perturbed DNA methylation.

A: Overview of the viral injection protocol into the SNpc of wild-type mice. Wild-type $m$ Cherry or mouse Gadd45b with its regulatory sequences under the control of the Ef1a promoter. Animals were then sacrificed 14 or 90 days later to perform RRBS, immunostainings or RT-qPCRs.

B: The AAV8-mCherry virus diffuses within the SNpc and infects mDA neurons. injected mice showing the diffusion of the virus in the SNpc area while sparing the VTA. Scale bar represents $300 \mu \mathrm{m}$. Lower panel: ISH of Gadd45b expression. Gadd45b expression in the SNpc of AAV8-mGadd45b injected mice at 14d p.i. shows that Gadd45b is overexpressed only in the ipsilateral SNpc and not in the ipsilateral VTA, nor on the contralateral side.

C: Exogenous Gadd45b RNA is expressed 14d after the injection of AAV8mGadd45b. Transcript expression analysis by RT-qPCR following manual microdissection of the SNpc of the injected side (upper panel) show a 79-fold increase in mGadd45B transcript level (mCherry= 11.02 \pm 4.83 ; mGadd45B= $869.10 \pm 282.80$; mean \pm SEM) at 14d p.i. (left); $n=6$; and a 178 fold increase

D-I: RRBS analysis of differentially methylated CpGs (DMCs) in the SNpc region after injection of AAV8-mGadd45b or AAV8-control.

D: Volcano plot of differentially methylated CpGs shows widespread perturbations in DNA methylation upon Gadd45b overexpression 14d p.i.. 76185 significantly differentially methylated CpGs were detected with a q-value smaller than or equal to

8520.01 and at least 25\% difference. The Volcano plot shows the number of regions with changed patterns of methylation between Gadd45b and control samples significantly higher or lower than the $25 \%$ difference cut-off and considering a q-value threshold of 0.01. The difference in methylation is reflected in the $x$-axis while the $y$-axis represents the significance of the difference. Regions that are highly differentially methylated are further to the left and right sides of the plot, while highly significant 858 changes appear higher on the plot. Values on the $x$ - and $y$-axes are percent 
859

860

861

862

863

864

865

866

867

868

869

870

871

872

873

874

875

876

877

878

879

880

881

882

883

884

885

886

887

888

889

890

891

methylation differences and negative log10 of the corrected p-values, respectively. The pie chart shows the percentage of hyper- and hypomethylated regions.

$\boldsymbol{E}$ and $\boldsymbol{F}$ : DMCs are located majorly in open sea regions and in introns of genes. $D M C s$ were annotated in relation to the distance to a $C p G$ island $(E)$, as well as based on the genomic regions they are associated with $(\boldsymbol{F})$. Their distributions are plotted in a bar chart.

G: Comparison of total number, common genetic intervals (minimum overlap of $1 \mathrm{bp}$ ) and percentage in common for DMCs and DMRs 14d and 90d p.i..

$\boldsymbol{H}$ : The "Gadd45b-DMC-regulon". Venn diagrams showing the overlap of genes containing at least one intronic hypomethylated DMC at 14 and 90d (4188 genes, left upper Venn diagram), overlap of genes containing at least one hypermethylated DMC at 14d and 90d (3827 genes, right upper Venn diagram) and the overlap of both groups (2353 genes, lower Venn diagram) representing the "Gadd45b-DMCregulon". Note that the "Gadd45b-DMR-regulon" encomprising 447 genes is summarized in the Suppl. Fig.1E.

I: GO-analysis of the "Gadd45b-DMC-regulon" reveals neuron-related gene categories enriched after Gadd45b overexpression 14d p.i.. Gene ontology analysis (PANTHER version 15.0) and the PANTHER overrepresentation test with the GOSlim annotation data set 'biological process' identified significantly overrepresented GO categories. The first fifteen significantly overrepresented GO categories with the highest fold enrichment are displayed with the fold enrichment on the left $y$-axis (black points) and the FDR value on the right y-axis (grey points).

Figure 2. Gadd45b overexpression leads to heterochromatin destructuration in mDA neurons.

A-E: H3K9me3 heterochromatin staining is perturbed as early as $14 d$ after the injection of AAV8-mGadd4b. TH+ neurons in the SNpc of AAV8-mGadd45b injected mice $(\boldsymbol{A})$ display at $14 d$ p.i. a 1.13-fold increase in the number $(3.64 \pm 0.12 ; 4.10 \pm 0.12$, data represented as a frequency distribution histogramm in $\boldsymbol{B})$ and a 1.24-fold increase in the volume $\left(4.78 \pm 0.15 ; 5.91 \pm 0.22 \mu m^{3}\right.$, C) of H3K9me3 foci. The diffuse nucleoplasmic staining intensity $\left(2.37 \times 10^{7} \pm 557961 ; 1.96 \times 10^{7} \pm 441292\right)$ is decreased by 1.21-fold (D) while the foci intensity $\left(1.32 \times 10^{6} \pm 54236\right.$; $\left.1.41 \times 10^{6} \pm 64942\right)$ remains unchanged (E). 
892

893

894

895

896

897

898

899

900

901

902

903

904

905

906

907

908

909

910

911

912

913

914

915

916

917

918

919

920

921

922

923

924

F-J: H3K9me3 heterochromatin staining remains perturbed up until 90d after the injection of AAV8-mGadd45b. A similar pattern as in A-E was observed at $90 d$ p.i. with a 1.18-fold increase in the number (4.07 $\pm 0.17 ; 4.82 \pm 0.20$, data represented as a frequency distribution histogramm in $\mathbf{G )}$ and a 1.15-fold increase in the volume $\left(5.51 \pm 0.18 ; 6.35 \pm 0.24 \mu m^{3}, \boldsymbol{H}\right)$ of $H 3 K 9 m e 3$ foci shown in (F). The diffuse nucleoplasmic staining intensity $\left(5.66 \times 10^{7} \pm 5 \times 10^{6} ; 1.97 \times 10^{7} \pm 573406\right)$ is decreased by 2.87-fold while the foci intensity $\left(1.22 \times 10^{6} \pm 56943 ; 1.26 \times 10^{6} \pm 74064\right)$ remains unchanged (I; J). Scale bar in $\boldsymbol{A}$ and $\boldsymbol{F}$ represents $5 \mu \mathrm{m}$. ${ }^{* *} p<0.01 ;{ }^{* * *} p<0.001$; ${ }^{* * *}$ $p<0.0001 ; n=3$ mice; Between 510 and 534 neurons were quantified per condition at $14 d$ p.i. and 428 neurons were quantified per condition at 90d p.i.. Error bars represent SEM.

Figure 3. Loss of TH+ neurons and their increased vulnerability upon Gadd45b overexpression.

A-C: Dopaminergic cell loss in the SNpc 90d following the injection of AAV8$m G a d d 45 b$. The ratio of the number of $\mathrm{TH}+$ neurons of the injected side on the noninjected side is similar at $14 d$ p.i. between AAV8-mCherry and AAV8-mGadd4b injected mice and close to 1 (1.04 $\pm 0.02 ; 0.97 \pm 0.04)$ (B). At 90d p.i. however, if the ratio of AAV8-mCherry mice is still close to 1 (0.97 \pm 0.02$)$, the ratio of AAV8mGadd45b mice $(0.82 \pm 0.04)$ reflect a $18 \%$ loss of $\mathrm{TH}+$ neurons compared to the non-injected side, shown in (A) and quantified in (C); scale bar represents $1000 \mu \mathrm{m}$; $n=5$.

D-F: Dopaminergic neurons are sensitized to 6-OHDA upon Gadd45b overexpression. At 14d p.i., AAV8-mCherry and AAV8-mGadd45b mice were injected with 6-OHDA in the striatum, on the same side as the viral injection and analyzed 3 days after (Fig. 1A). TH staining of dopaminergic axons shows that axonal degeneration in the striatum induced by 6-OHDA occurs in AAV8-mCherry mice (0.71 \pm 0.04$)$ but is 1.5-fold more pronounced in AAV8-mGadd45b mice $(0.56 \pm 0.04)$ shown in (D), quantified in (E). The number of $\mathrm{TH}+$ neurons is unaffected (0.96 $\pm 0.04 ; 0.85 \pm 0.03)$ after the 3 day 6-OHDA treatment $(\boldsymbol{F}) ; n=4-6$; Scale bar represents $1000 \mu \mathrm{m}$.

G-J: Gadd45b expression accentuates heterochromatin loss and MeCP2 foci dispersion upon striatal 6-OHDA injection. TH+ neurons in the SNpc of AAV8- 
925 mGadd45b mice injected with 6-OHDA at 14d p.i. display 3 days after the 6-OHDA 926 injection an increase by 1.47 -fold in the number of $\mathrm{H} 3 \mathrm{~K} 9 \mathrm{me} 3$ foci $(3.28 \pm 0.15$; 927 4.82 \pm 0.16$)$, shown in (G), quantified and represented as a frequency distribution 928 histogramm in $(\boldsymbol{H})$ and by 1.16-fold in the number of MeCP2 foci $(2.97 \pm 0.12$; $9293.45 \pm 0.08)$, shown in (I), quantified and represented as a frequency distribution 930 histogramm in (J). Scale bar represents $5 \mu \mathrm{m} ;{ }^{*} p<0,05 ;{ }^{* *} p<0,01 ; * * * * 0<0,0001$; 931 n=3; Between 632 and 887 neurons were quantified per condition for H3K9me3 foci and between 94 and 276 neurons per condition for MeCP2 foci. Error bars represent SEM.

Figure 4. DNA methylation changes and heterochromatin de-structuration upon Gadd45b overexpression are associated with DNA damage.

937 A-C: Gadd45b overexpression induced DNA damage in TH+ neurons of the SNpc. 938 The majority (65.94 $2.10 \%)$ of $\mathrm{TH}+$ neurons of the SNpc in AAV8-mGadd45b display no single $\gamma-\mathrm{H} 2 \mathrm{AX}$ foci but instead an intense diffuse nucleoplasmic staining,

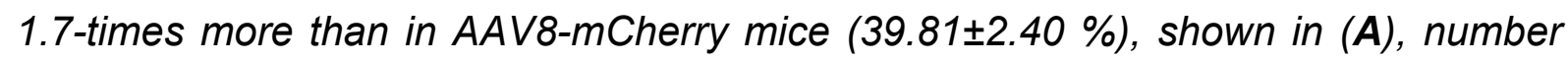
quantified in (B), diffuse nucleoplasmic intensity quantified and represented as a frequency distribution histogram in (C). Scale bar represents $5 \mu m ; n=3$. Between 417 and 518 neurons were quantified per condition.

944

Figure 5. LINE-1 methylation changes and increased LINE-1 expression 946 following Gadd45b overexpression.

947 A-C: Analysis of DMCs in LINE-1 sequences (L1-DMCS) 14d p.i. of AAV8948 mGadd45b.

949 A: Location of L1-DMCs. 22.5\% of all (3530 L1-DMCs are located in introns.

950 B: Of all the L1-DMCs at 14d p.i., most (54.7\%) are hypomethylated (blue).

951 C: The ten most represented L1 families with at least one L1-DMC.

952 D-G: Analysis of DMCs in intronic LINE-1 sequences (L1-iDMCs) 14d p.i. of AAV8953 mGadd45b.

954 Most L1-iDMCs (54.7\%) are hypomethylated (D), located in protein-coding genes (in 955483 genes, $\boldsymbol{E})$ and related to neuronal functions (F).

956 G: The ten most represented L1 families with L1-iDMCs. 
$\boldsymbol{H}$ : Analysis of the methylation status of full-length LINE-1 (fIL1) elements containing a DMC or a DMR. Most DMCs and DMRs in full-length $L 1$ elements are hypomethylated.

I: LINE-1 transcripts of the young $L 1 M d-T$ and $L 1 M d-A$ families are increased after injection of AAV8-mGadd45b compared to AAV8-mCherry 14d p.i.. RT-qPCR of RNA extracted from manually micro-dissected SNpc of the injected sides shows a 1.5-fold

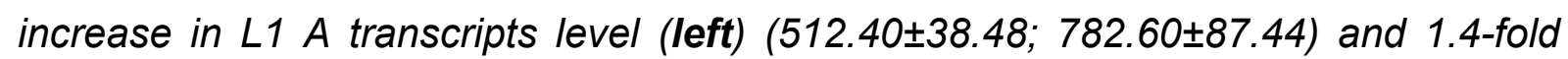

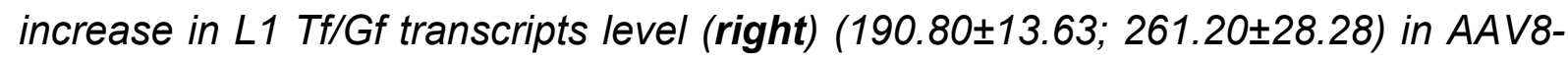
mGadd45B mice at $14 d$ p.i. compared to AAV8-mCherry mice; * $p<0,05$; ${ }^{* *} p<0,01$; ${ }^{* * *} p<0,0001 ; n=6$. Error bars represent SEM.

Figure 6: Candidate genes with DMCs upon Gadd45b overexpression show altered expression in the SNpc at 90d p.i.

A: The expression of several gene candidates containing DMCs is dysregulated. Setdb1, Park2 Dnmt3a, Tet3 and Satb1 transcripts are downregulated 90d p.i. of AAV8-mGadd45b. The mRNA analysis in SNpc of the injected side by RT-qPCR shows no significant difference in Satb1 (4.87 \pm 0.59 ; 5.57 \pm 0.65$)$, Setdb1 (9.03 \pm 0.76 ; 7.51 \pm 0.42$)$, Dnmt3a (24.07 $\pm 2.52 ; 22.76 \pm 0.92)$, Tet3 (2.16 $\pm 0.26 ; 2.20 \pm 0.65)$ and Park2 (1.46 $\pm 0.21 ; 1.65 \pm 0.09)$ transcripts at $14 d$ p.i. (4.87 $\pm 0.59 ; 5.57 \pm 0.65) ; n=5-6$. At 90d p.i., there is a decrease in transcript levels of 1.9-fold for Satb1 (4.43 \pm 0.30 ;

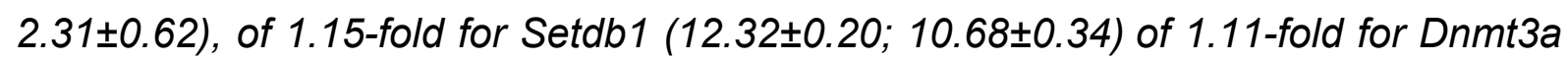
(30.06 $\pm 0.47 ; 27.05 \pm 0.67)$, of 1.14 fold for Tet3 $(3.89 \pm 0.09 ; 3.40 \pm 0.17)$ of 1.25 -fold for Park2 (3.42 $\pm 0.12 ; 2.73 \pm 0.18)$, in AAV8-mGadd45b mice compared to AAV8$m$ Cherry mice; * $p<0,05 ; n=4$. Error bars represent SEM.

B: Genes significantly deregulated upon Gadd45b overexpression were analyzed with regard to methylation differences throughout their gene bodies (exonic and intronic DMCs) at 14d and 90d p.i.. Results are displayed for Satb1, Setdb1, Dnmt3a, Tet3 and Park2. Exact chromosomal positions of DMCs are given on the $x$-axis and the orientation of the genes is indicated by an arrow. Hypermethylated are depicted as red squares while hypomethylated DMCs are depicted in blue squares. DMCs were defined as having a methylation difference > or equal to 25 (dashed line) and a $q$-value $<0.01$ 
bioRxiv preprint doi: $h t t p s: / / d o i . o r g / 10.1101 / 2020.06 .23 .158014$; this version posted June 23, 2020. The copyright holder for this preprint

(which was not certified by peer review) is the author/funder, who has granted bioRxiv a license to display the preprint in perpetuity. It is made available under aCC-BY-NC-ND 4.0 International license.

990 Table 1. Summary of the differential methylation analysis comparing Gadd45b

991 overexpression to mCherry control in the SNpc of wild-type mice. 
993

994

995

996

997

998

999

1000

1001

1002

1003

1004

1005

1006

1007

1008

1009

1010

1011

1012

1013

1014

1015

1016

1017

1018

1019

1020

1021

1022

1023

1024

1025

1026

1027

1028

1029

1030

1031

1032

1033

1034

1035

1036

1037

1038

\section{References}

1. Solovei, I., Thanisch, K. \& Feodorova, Y. How to rule the nucleus: divide et impera. Curr. Opin. Cell Biol. 40, 47-59 (2016).

2. Benayoun, B. A., Pollina, E. A. \& Brunet, A. Epigenetic regulation of ageing: linking environmental inputs to genomic stability. Nat. Rev. Mol. Cell Biol. 16, 593-610 (2015).

3. Pal, S. \& Tyler, J. K. Epigenetics and aging. Sci. Adv. 2, e1600584-19 (2016).

4. Lodato, M. A. et al. Aging and neurodegeneration are associated with increased mutations in single human neurons. Science (2017).

doi:10.1126/science.aao4426

5. Burgess, R. C., Misteli, T. \& Oberdoerffer, P. DNA damage, chromatin, and transcription: the trinity of aging. Curr. Opin. Cell Biol. 24, 724-730 (2012).

6. Lander, E. S. et al. Initial sequencing and analysis of the human genome. Nature 409, 860-921 (2001).

7. Mouse Genome Sequencing Consortium et al. Initial sequencing and comparative analysis of the mouse genome. Nature 420, 520-562 (2002).

8. Trelogan, S. A. \& Martin, S. L. Tightly regulated, developmentally specific expression of the first open reading frame from LINE-1 during mouse embryogenesis. Proc. Natl. Acad. Sci. U.S.A. 92, 1520-1524 (1995).

9. Wei, W. et al. Human L1 retrotransposition: cis preference versus trans complementation. Molecular and Cellular Biology 21, 1429-1439 (2001).

10. Martin, S. L. \& Bushman, F. D. Nucleic acid chaperone activity of the ORF1 protein from the mouse LINE-1 retrotransposon. Molecular and Cellular Biology 21, 467-475 (2001).

11. Hattori, M., Kuhara, S., Takenaka, O. \& Sakaki, Y. L1 family of repetitive DNA sequences in primates may be derived from a sequence encoding a reverse transcriptase-related protein. Nature 321, 625-628 (1986).

12. Feng, Q., Moran, J. V., Kazazian, H. H. \& Boeke, J. D. Human L1 retrotransposon encodes a conserved endonuclease required for retrotransposition. Cell 87, 905-916 (1996).

13. Pizarro, J. G. \& Cristofari, G. Post-Transcriptional Control of LINE-1 Retrotransposition by Cellular Host Factors in Somatic Cells. Front Cell Dev Biol 4, 14 (2016).

14. Van Meter, M. et al. SIRT6 represses LINE1 retrotransposons by ribosylating KAP1 but this repression fails with stress and age. Nat Commun 5, 5011 (2014).

15. Kazazian, H. H. \& Goodier, J. L. LINE drive. retrotransposition and genome instability. Cell 110, 277-280 (2002).

16. Gasior, S. L., Wakeman, T. P., Xu, B. \& Deininger, P. L. The human LINE-1 retrotransposon creates DNA double-strand breaks. J. Mol. Biol. 357, 13831393 (2006).

17. Simon, M. et al. LINE1 Derepression in Aged Wild-Type and SIRT6- Deficient Mice Drives Inflammation. Cell Metab. 1-21 (2019). doi:10.1016/j.cmet.2019.02.014

18. Blaudin de Thé, F.-X. et al. Engrailed homeoprotein blocks degeneration in adult dopaminergic neurons through LINE-1 repression. EMBO J 37, (2018). 
1039

1040

1041

1042

1043

1044

1045

1046

1047

1048

1049

1050

1051

1052

1053

1054

1055

1056

1057

1058

1059

1060

1061

1062

1063

1064

1065

1066

1067

1068

1069

1070

1071

1072

1073

1074

1075

1076

1077

1078

1079

1080

1081

1082

1083

1084

1085

1086

1087

1088

19. Collier, T. J., Kanaan, N. M. \& Kordower, J. H. Ageing as a primary risk factor for Parkinson's disease: evidence from studies of non-human primates. Nat Rev Neurosci 12, 359-366 (2011).

20. López-Otín, C., Blasco, M. A., Partridge, L., Serrano, M. \& Kroemer, G. The hallmarks of aging. Cell 153, 1194-1217 (2013).

21. Gan, L., Cookson, M. R., Petrucelli, L. \& La Spada, A. R. Converging pathways in neurodegeneration, from genetics to mechanisms. Nat Neurosci 21, 13001309 (2018).

22. Hou, Y. et al. Ageing as a risk factor for neurodegenerative disease. Nat Rev Neurol 15, 565-581 (2019).

23. Fasolino, M., Liu, S., Wang, Y. \& Zhou, Z. Distinct cellular and molecular environments support aging-related DNA methylation changes in the substantia nigra. Epigenomics 9, 21-31 (2017).

24. Sanchez-Mut, J. V. et al. Human DNA methylomes of neurodegenerative diseases show common epigenomic patterns. 6, e718-8 (2016).

25. Labbé, C., Lorenzo-Betancor, O. \& Ross, O. A. Epigenetic regulation in Parkinson's disease. Acta Neuropathol 132, 515-530 (2016).

26. Park, G. et al. Regulation of Histone Acetylation by Autophagy in Parkinson Disease. Journal of Biological Chemistry 291, 3531-3540 (2016).

27. Gavin, D. P. et al. Gadd45b and N-methyl-D-aspartate induced DNA demethylation in postmitotic neurons. Epigenomics 7, 567-579 (2015).

28. Niehrs, C. \& Schäfer, A. Active DNA demethylation by Gadd45 and DNA repair. Trends Cell Biol. 22, 220-227 (2012).

29. Ma, D. K. et al. Neuronal Activity-Induced Gadd45b Promotes Epigenetic DNA Demethylation and Adult Neurogenesis. Science 323, 1074-1077 (2009).

30. Rekaik, H. et al. Engrailed Homeoprotein Protects Mesencephalic Dopaminergic Neurons from Oxidative Stress. CellReports 13, 242-250 (2015).

31. Chen, K. et al. Gadd45a is a heterochromatin relaxer that enhances iPS cell generation. EMBO reports 17, 1641-1656 (2016).

32. Blandini, F., Armentero, M.-T. \& Martignoni, E. The 6-hydroxydopamine model: news from the past. Parkinsonism and realted Disorders 14 Suppl 2, S124-9 (2008).

33. Bagga, V., Dunnett, S. B. \& Fricker, R. A. The 6-OHDA mouse model of Parkinson's disease - Terminal striatal lesions provide a superior measure of neuronal loss and replacement than median forebrain bundle lesions. Behav. Brain Res. 288, 107-117 (2015).

34. Villeponteau, B. The heterochromatin loss model of aging. Exp. Gerontol. 32, 383-394 (1997).

35. Peters, A. H. et al. Loss of the Suv39h histone methyltransferases impairs mammalian heterochromatin and genome stability. Cell 107, 323-337 (2001).

36. Pegoraro, G. et al. Ageing-related chromatin defects through loss of the NURD complex. Nature Cell Biology 11, 1261-1267 (2009).

37. Sun, W., Samimi, H., Gamez, M., Zare, H. \& Frost, B. Pathogenic tau-induced piRNA depletion promotes neuronal death through transposable element dysregulation in neurodegenerative tauopathies. Nat Neurosci 21, 1038-1048 (2018).

38. Frost, B., Hemberg, M., Lewis, J. \& Feany, M. B. Tau promotes neurodegeneration through global chromatin relaxation. Nat Neurosci 17, 357366 (2014). 
1089

1090

1091

1092

1093

1094

1095

1096

1097

1098

1099

1100

1101

1102

1103

1104

1105

1106

1107

1108

1109

1110

1111

1112

1113

1114

1115

1116

1117

1118

1119

1120

1121

1122

1123

1124

1125

1126

1127

1128

1129

1130

1131

1132

1133

1134

1135

1136

1137

1138

39. Hajjar, El, J. et al. Heterochromatic genome instability and neurodegeneration sharing similarities with Alzheimer's disease in old Bmi1+/- mice. Sci. Rep. 116 (2019). doi:10.1038/s41598-018-37444-3

40. Belgnaoui, S. M., Gosden, R. G., Semmes, O. J. \& Haoudi, A. Human LINE-1 retrotransposon induces DNA damage and apoptosis in cancer cells. Cancer Cell Int. 6, 13 (2006).

41. Sookdeo, A., Hepp, C. M., McClure, M. A. \& Boissinot, S. Revisiting the evolution of mouse LINE-1 in the genomic era. Mob DNA 4, 1-1 (2013).

42. Penzkofer, T. et al. L1Base 2: more retrotransposition-active LINE-1s, more mammalian genomes. Nucleic Acids Research 45, D68-D73 (2017).

43. Blaudin de Thé, F.-X., Rekaik, H., Prochiantz, A., Fuchs, J. \& Joshi, R. L. Neuroprotective Transcription Factors in Animal Models of Parkinson Disease. Neural Plast. 2016, 6097107 (2016).

44. $\mathrm{Li}$, Z. et al. Gadd45a promotes DNA demethylation through TDG. Nucleic Acids Research 43, 3986-3997 (2015).

45. Labonté, B. et al. Gadd45b mediates depressive-like role through DNA demethylation. Sci. Rep. 9, 4615 (2019).

46. Gavin, D. P. et al. Growth arrest and DNA-damage-inducible, beta (GADD45b)mediated DNA demethylation in major psychosis. Neuropsychopharmacology 37, 531-542 (2012).

47. Apulei, J. et al. Non-cell Autonomous OTX2 Homeoprotein Regulates Visual Cortex Plasticity Through Gadd45b/g. Cereb. Cortex 29, 2384-2395 (2019).

48. Kienhöfer, S. et al. GADD45a physically and functionally interacts with TET1. Differentiation 90, 59-68 (2015).

49. Arab, K. et al. GADD45A binds R-loops and recruits TET1 to CpG island promoters. Nat Genet 1-13 (2018). doi:10.1038/s41588-018-0306-6

50. Bayraktar, G. \& Kreutz, M. R. The Role of Activity-Dependent DNA Demethylation in the Adult Brain and in Neurological Disorders. Front Mol Neurosci 11, 169 (2018).

51. Lee, M. et al. Epigenomic analysis of Parkinson's disease neurons identifies \&lt;em\&gt;Tet2\&lt;/em\&gt; loss as neuroprotective. bioRxiv 779785 (2019).

52. Perera, A. et al. TET3 Is Recruited by REST for Context-Specific Hydroxymethylation and Induction of Gene Expression. CellReports 11, 283294 (2015).

53. Jin, S.-G. et al. Tet3 Reads 5-Carboxylcytosine through Its CXXC Domain and Is a Potential Guardian against Neurodegeneration. CellReports 14, 493-505 (2016).

54. Chuang, Y.-H. et al. Longitudinal Epigenome-Wide Methylation Study of Cognitive Decline and Motor Progression in Parkinson's Disease. J Parkinsons Dis 9, 389-400 (2019).

55. Pihlstrøm, L., Berge, V., Rengmark, A. \& Toft, M. Parkinson's disease correlates with promoter methylation in the $\alpha$-synuclein gene. Mov Disord. 30, 577-580 (2015).

56. Wüllner, U., Kaut, O., deBoni, L., Piston, D. \& Schmitt, I. DNA methylation in Parkinson's disease. J Neurochem 139 Suppl 1, 108-120 (2016).

57. $\mathrm{Li}, \mathrm{P}$. et al. Epigenetic dysregulation of enhancers in neurons is associated with Alzheimerâ $€^{\mathrm{TM}} \mathbf{s}$ disease pathology and cognitive symptoms. Nat Commun 114 (2019). doi:10.1038/s41467-019-10101-7

58. Nativio, R. et al. Dysregulation of the epigenetic landscape of normal aging in Alzheimer's disease. Nat Neurosci 21, 497-505 (2018). 
59. Berson, A., Nativio, R., Berger, S. L. \& Bonini, N. M. Epigenetic Regulation in Neurodegenerative Diseases. Trends in Neurosciences 41, 587-598 (2018).

60. Cedar, H. \& Bergman, Y. Linking DNA methylation and histone modification: patterns and paradigms. Nature Reviews Genetics 10, 295-304 (2009).

61. Schäfer, A. Gadd45 proteins: key players of repair-mediated DNA demethylation. Adv. Exp. Med. Biol. 793, 35-50 (2013).

62. Suberbielle, E. et al. Physiologic brain activity causes DNA double-strand breaks in neurons, with exacerbation by amyloid- $\beta$. Nature Publishing Group (2013). doi:10.1038/nn.3356

63. Simon, M. et al. Inhibition of retrotransposition improves health and extends lifespan of SIRT6 knockout mice. 1-53 (2018). doi:10.1101/460808

64. De Cecco, M. et al. Transposable elements become active and mobile in the genomes of aging mammalian somatic tissues. Aging (Albany NY) 5, 867-883 (2013).

65. Li, W. et al. Activation of transposable elements during aging and neuronal decline in Drosophila. Nature Publishing Group (2013). doi:10.1038/nn.3368

66. Wood, J. G. et al. Chromatin-modifying genetic interventions suppress ageassociated transposable element activation and extend life span in Drosophila. Proceedings of the National Academy of Sciences 201604621 (2016). doi:10.1073/pnas.1604621113

67. Liu, E. Y. et al. Loss of Nuclear TDP-43 Is Associated with Decondensation of LINE Retrotransposons. CellReports 27, 1409-1421.e6 (2019).

68. Billingsley, K. J. et al. Analysis of repetitive element expression in the blood and skin of patients with Parkinson's disease identifies differential expression of satellite elements. Sci. Rep. 9, 4369 (2019).

69. Li, W., Jin, Y., Prazak, L., Hammell, M. \& Dubnau, J. Transposable elements in TDP-43-mediated neurodegenerative disorders. PLoS ONE 7, e44099 (2012).

70. Södersten, E. et al. A comprehensive map coupling histonemodifications with gene regulation in adultdopaminergic and serotonergic neurons. Nat Commun 1-16 (2018). doi:10.1038/s41467-018-03538-9

71. Zahn, J. M. et al. AGEMAP: a gene expression database for aging in mice. PLoS Genet 3, e201 (2007).

72. Urdinguio, R. G., Sanchez-Mut, J. V. \& Esteller, M. Epigenetic mechanisms in neurological diseases: genes, syndromes, and therapies. Lancet Neurol 8, 1056-1072 (2009).

73. Hodges, A. et al. Regional and cellular gene expression changes in human Huntington's disease brain. Human Molecular Genetics 15, 965-977 (2006).

74. Narayanan, M. et al. Common dysregulation network in the human prefrontal cortex underlies two neurodegenerative diseases. Mol. Syst. Biol. 10, 743-743 (2014).

75. Prudencio, M. et al. Distinct brain transcriptome profiles in C9orf72-associated and sporadic ALS. Nat Neurosci 18, 1175-1182 (2015).

76. Soukup, S.-F. \& Verstreken, P. PIWIL1 protein power targets tau therapy. Nat Neurosci 17, 334-335 (2014).

77. Soukup, S.-F., Vanhauwaert, R. \& Verstreken, P. Parkinson's disease: convergence on synaptic homeostasis. EMBO J (2018). doi:10.15252/embj.201898960

78. Burke, R. E. \& O'Malley, K. Axon degeneration in Parkinson's disease. Experimental Neurology 246, 72-83 (2013). 
79. Jones, P. A. Functions of DNA methylation: islands, start sites, gene bodies and beyond. Nature Reviews Genetics 13, 484-492 (2012).

80. Kinde, B., Gabel, H. W., Gilbert, C. S., Griffith, E. C. \& Greenberg, M. E. Reading the unique DNA methylation landscape of the brain: Non-CpG methylation, hydroxymethylation, and MeCP2. Proceedings of the National Academy of Sciences 112, 201411269 (2015).

81. Neri, F. et al. Intragenic DNA methylation prevents spurious transcription initiation. Nature 1-30 (2017). doi:10.1038/nature21373

82. Yang, X. et al. Gene body methylation can alter gene expression and is a therapeutic target in cancer. Cancer Cell 26, 577-590 (2014).

83. Cancio-Bello, A. \& Saez-Atienzar, S. SATB1 is a dopaminergic neuron-specific regulator of cellular senescence. Mov Disord. 35, 235-235 (2020).

84. Riessland, M. et al. Loss of SATB1 Induces p21-Dependent Cellular Senescence in Post-mitotic Dopaminergic Neurons. Cell Stem Cell 25, 514530.e8 (2019).

85. Yasui, D., Miyano, M., Cai, S., Varga-Weisz, P. \& Kohwi-Shigematsu, T. SATB1 targets chromatin remodelling to regulate genes over long distances. Nature 419, 641-645 (2002).

86. Cai, S., Han, H.-J. \& Kohwi-Shigematsu, T. Tissue-specific nuclear architecture and gene expression regulated by SATB1. Nat Genet 34, 42-51 (2003).

87. Zhang, $X$. et al. DNMT3A and TET2 compete and cooperate to repress lineage-specific transcription factors in hematopoietic stem cells. Nat Genet 48, 1014-1023 (2016).

88. Krueger, F. \& Andrews, S. R. Bismark: a flexible aligner and methylation caller for Bisulfite-Seq applications. Bioinformatics 27, 1571-1572 (2011).

89. Akalin, A. et al. methylKit: a comprehensive R package for the analysis of genome-wide DNA methylation profiles. Genome Biol 13, R87 (2012).

90. Cavalcante, R. G. \& Sartor, M. A. annotatr: genomic regions in context. Bioinformatics 33, 2381-2383 (2017). 


\section{Ef1a mCherry AAV8-mCherry}

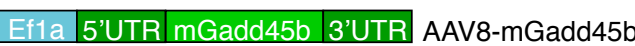

WT OF1 mice

Stereotaxic

injection $S N p c$

$\begin{array}{lrll} & & \text { L-OHDA } \\ \text { Day 0 } & \text { Day 14 } & +3 d & \text { Day 90 } \\ \text { RRBS } & \text { IHC/IF } & \text { RRBS } \\ \text { IHC/IF } & & \text { IHC/IF } \\ \text { RTgPCR } & & \text { RTgPCR }\end{array}$

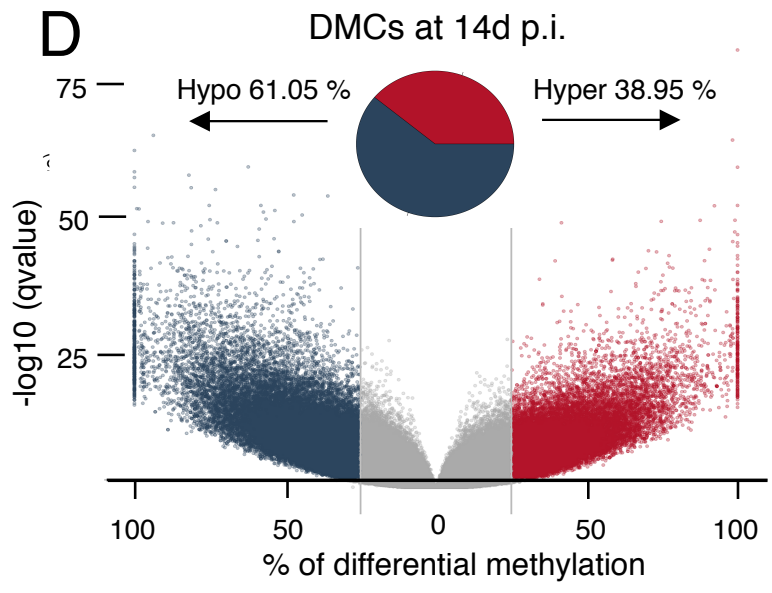

G

\begin{tabular}{|ccc|}
\hline & DMC & DMR \\
\hline Gadd45b vs Ctrl 14d p.i. & 76185 & 16702 \\
\hline Gadd45b vs Ctrl 90d p.i. & 76639 & 15685 \\
\hline Common genomic intervals & 13920 & 2517 \\
\hline Percentage in common (\%) & 18 & 15 \\
\hline
\end{tabular}
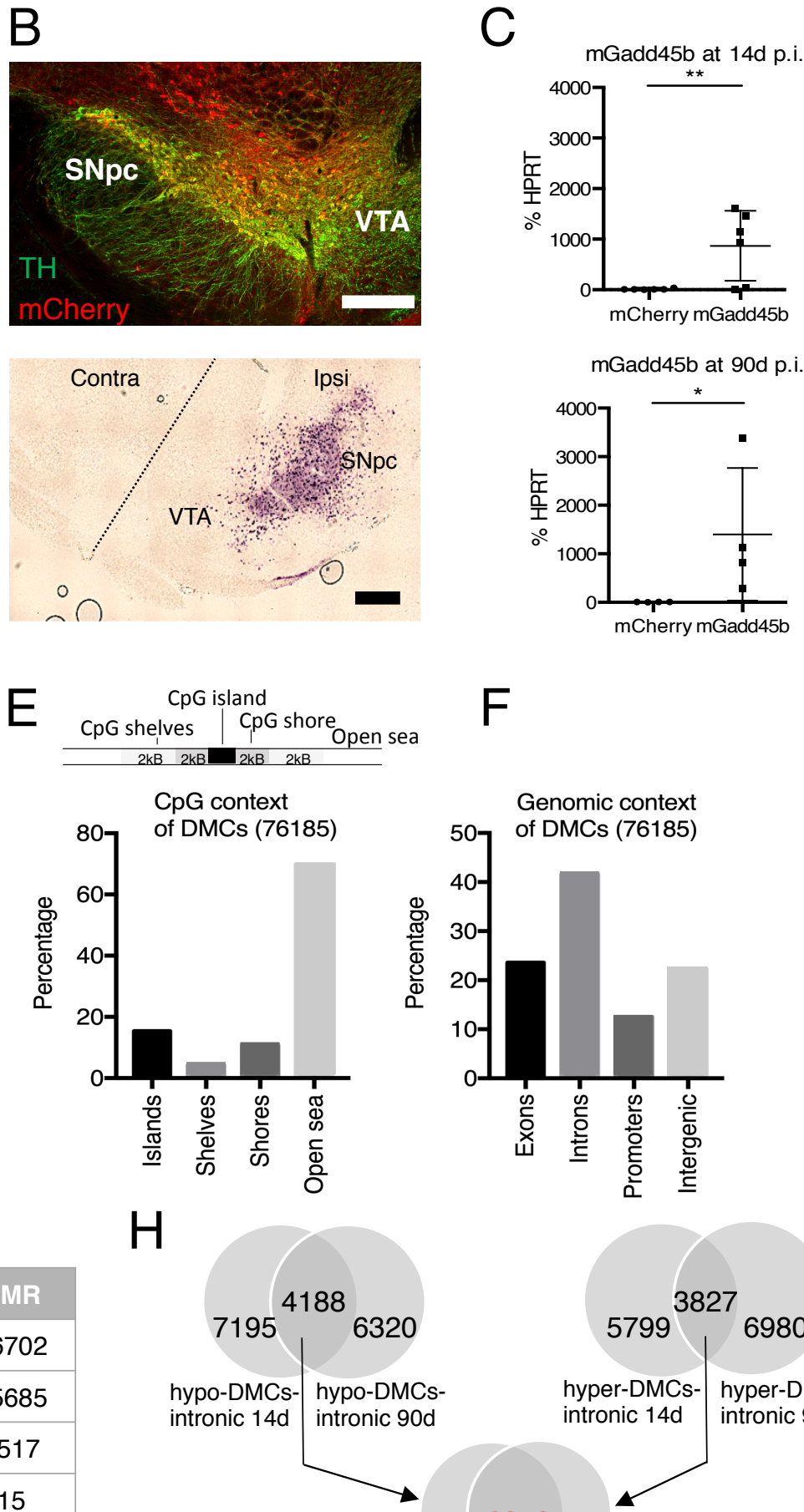

F Open sea

Fold enrichment

\section{3}
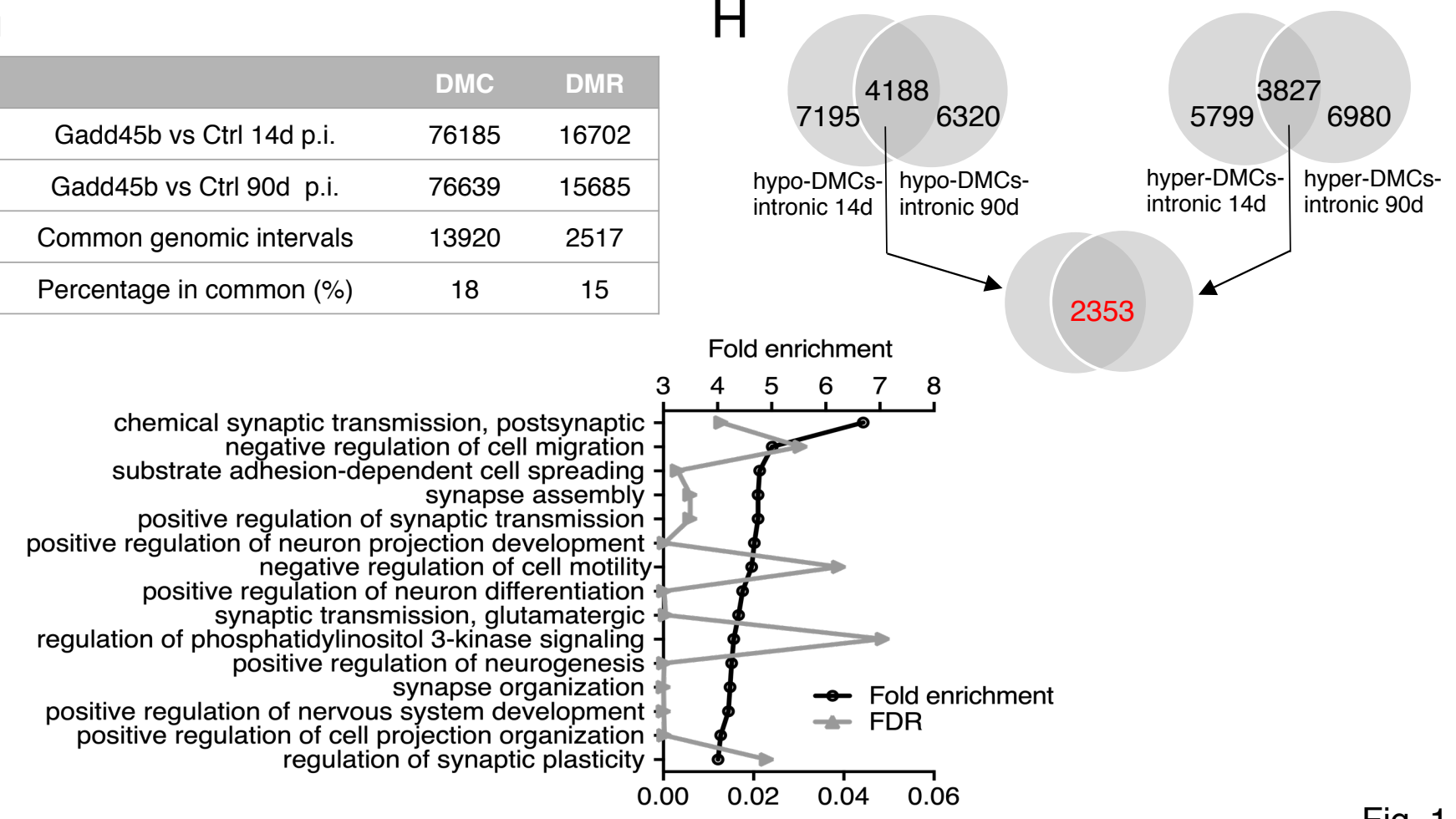

FDR

Fig. 1 


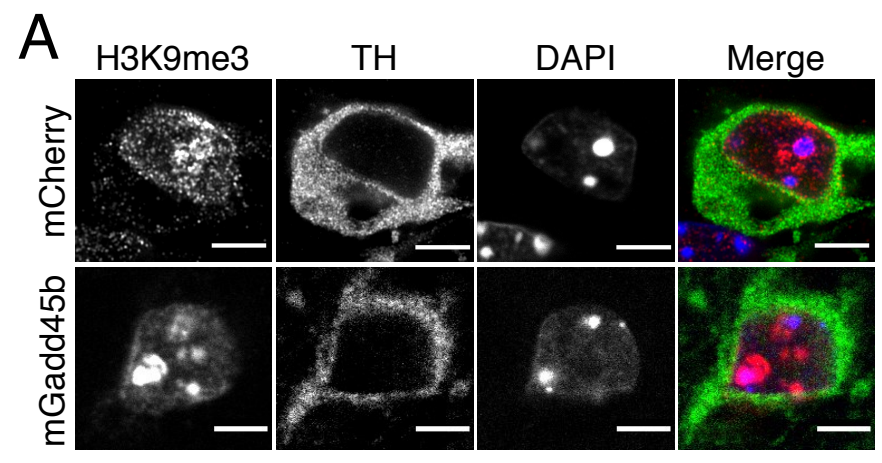

$\mathrm{C}_{\text {Volume of H3K9me3 foci } 14 \mathrm{~d} \text { p.i. }}$

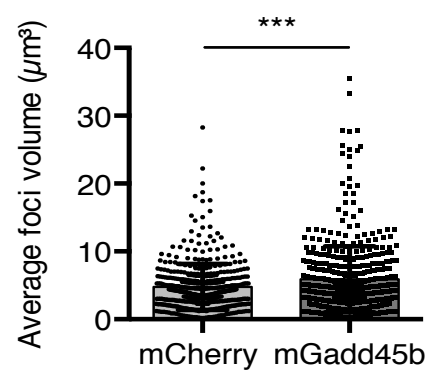

D Diffused H3K9me3 nucleus intensity 14d p.i.

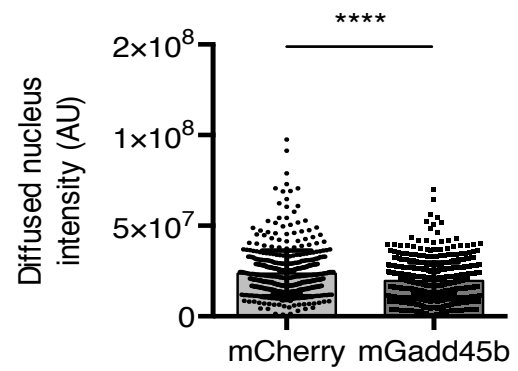

H3K9me3 foci at $14 d$ p.i.

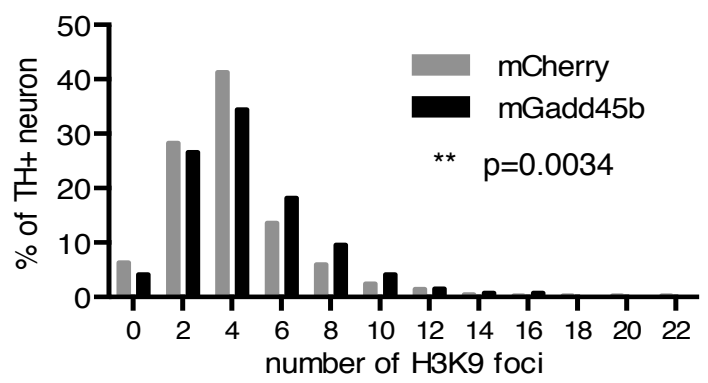

E

$\mathrm{F}$

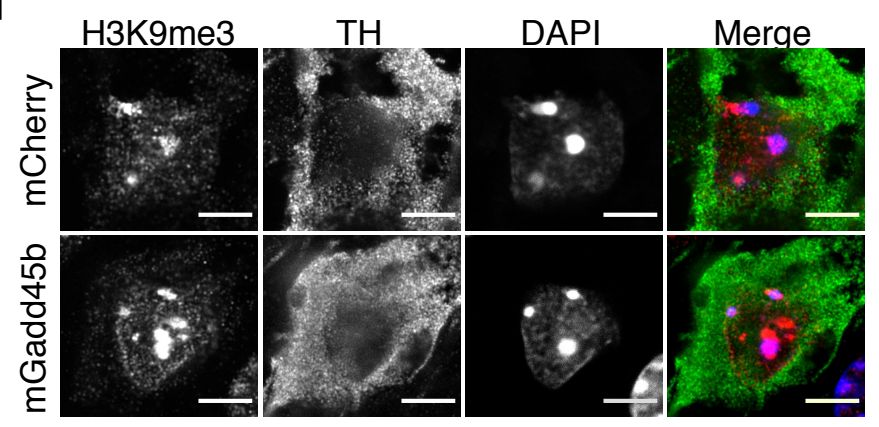

G H3K9me3 foci at $90 \mathrm{~d}$ p.i.

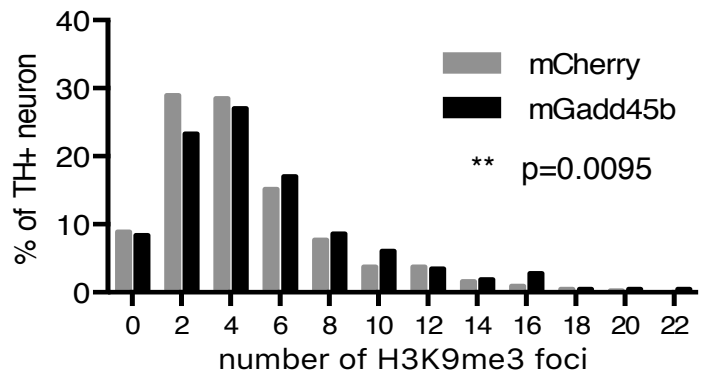

$\mathrm{H}_{\text {Volume of H3K9me3 foci } 90 d \text { p.i. }}$

Diffused H3K9me3 nucleus intensity 90d p.i. J

Intensity of H3K9me3 foci 90d p.i
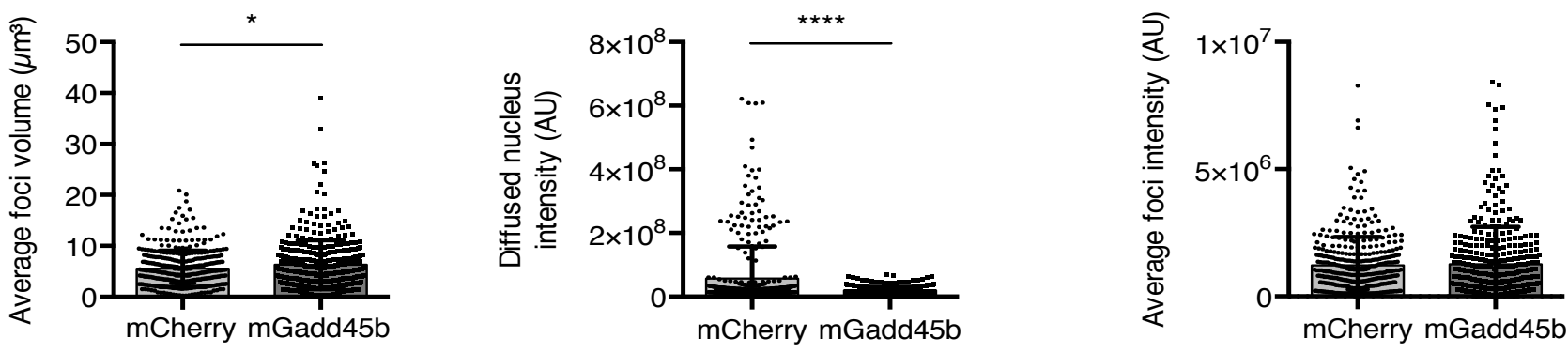

Fig. 2 
A
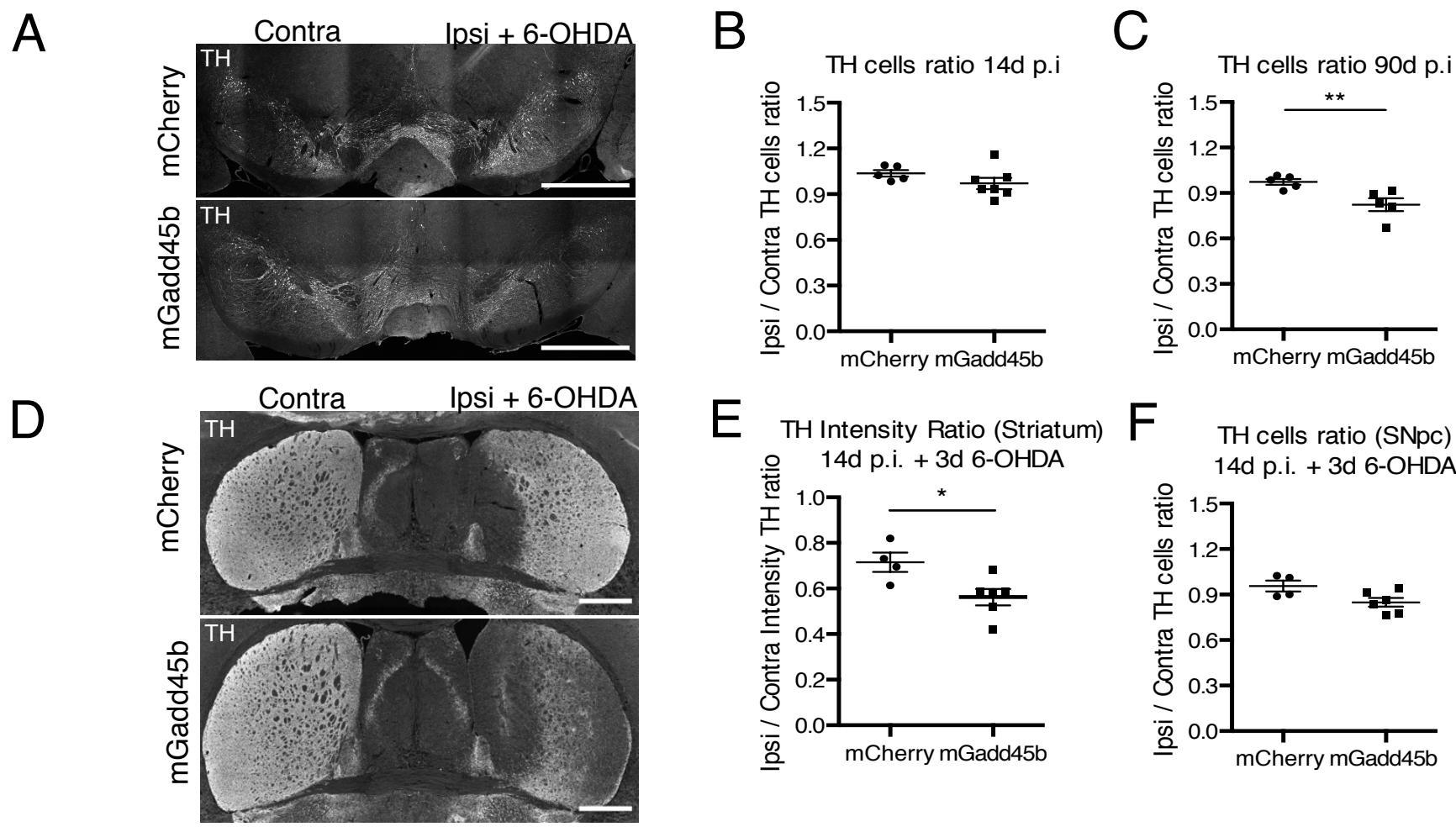

E TH Intensity Ratio (Striatum) F

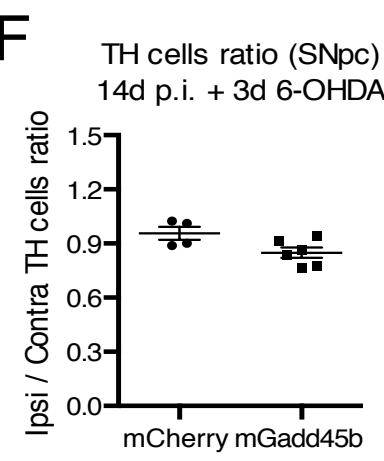

G
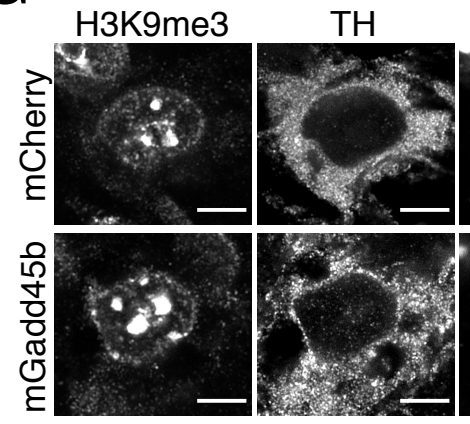

DAPI

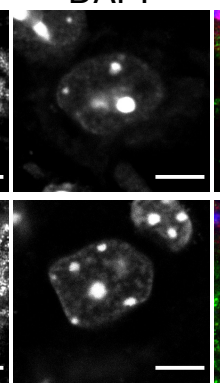

Merge

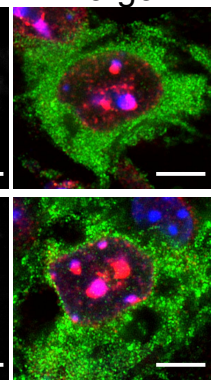

I
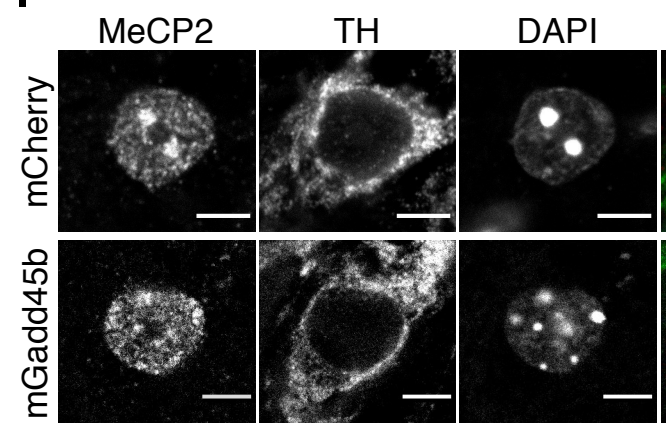

Merge
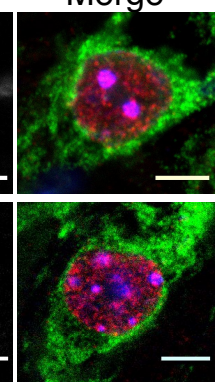

H3K9me3 foci at 14d p.i. + 3d 6-OHDA

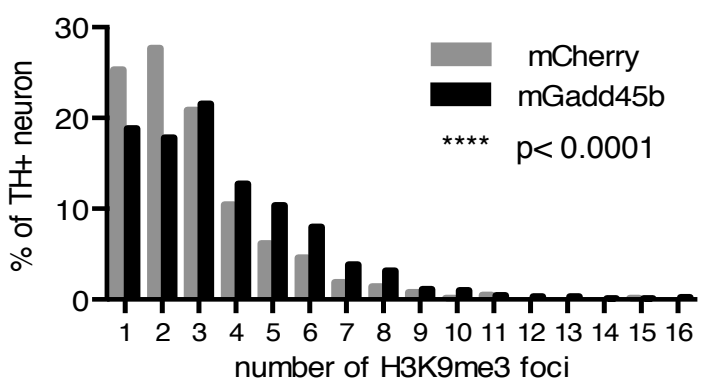

J

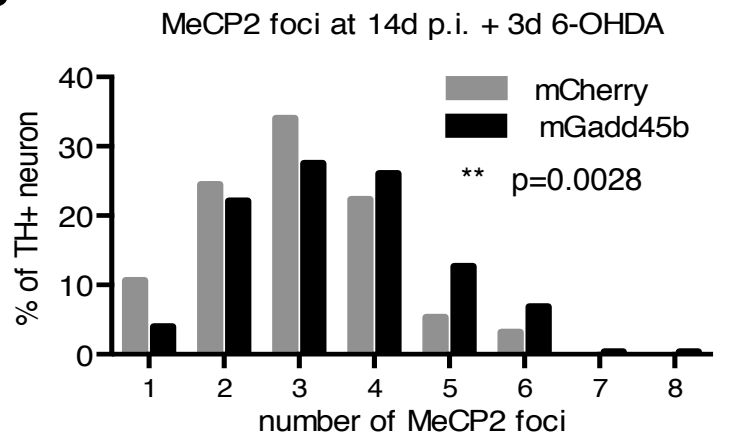


A

B

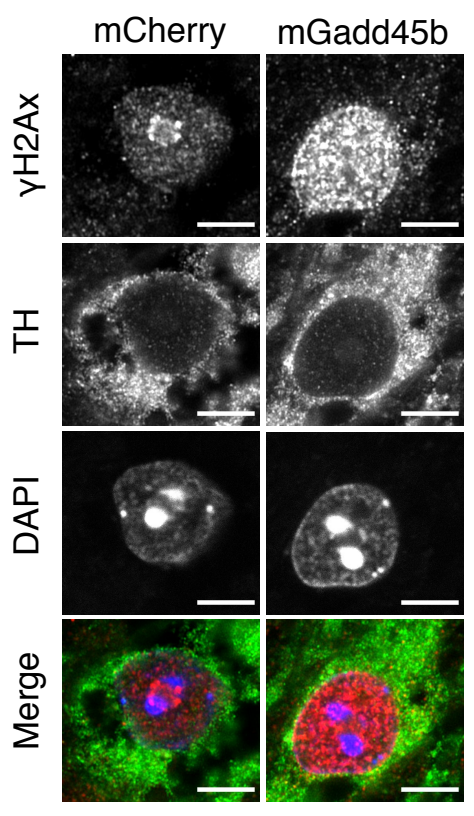

YH2Ax foci at $14 d$ p.i.

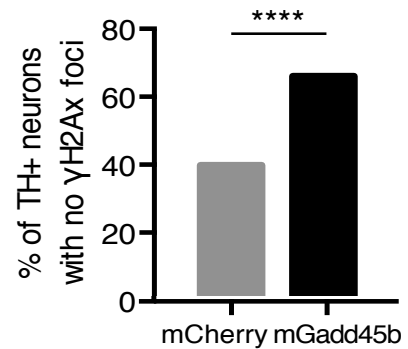

C Intensity of diffused nucleic $\mathrm{yH} 2 \mathrm{Ax}$ staining at $14 \mathrm{~d}$ p.i.

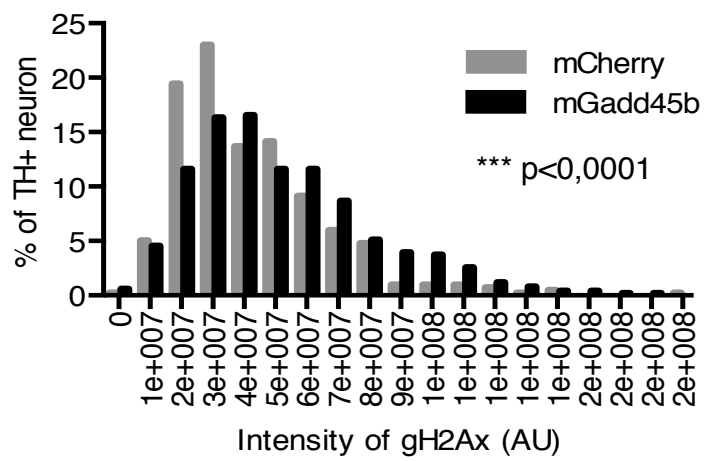

Fig. 4 
A

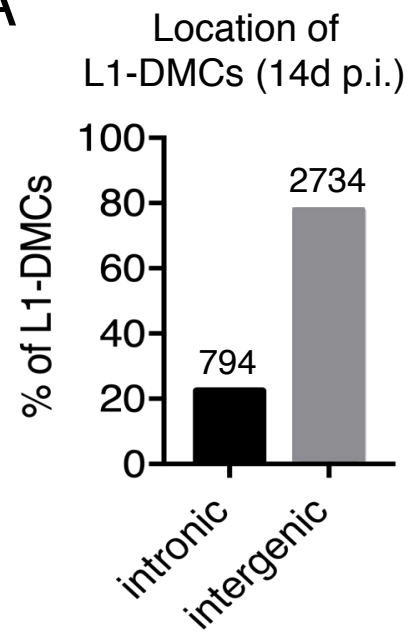

D Methylation status of L1-iDMCs (14d p.i.)

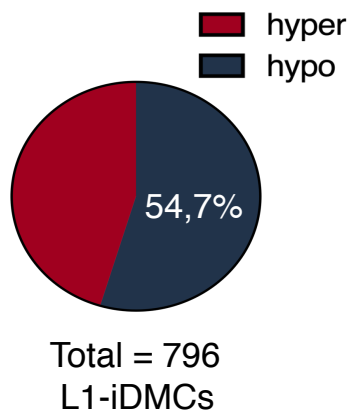

G GO analysis of genes containing a L1-iDMC

E Location of L1-iDMCs (14d p.i.)

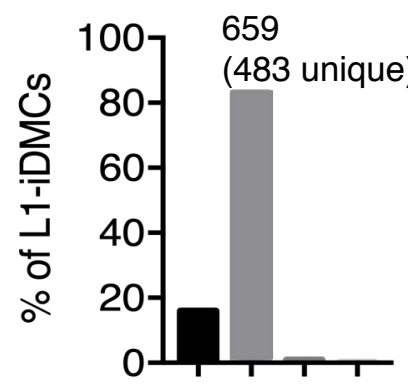

Total $=3530$

L1-DMCs
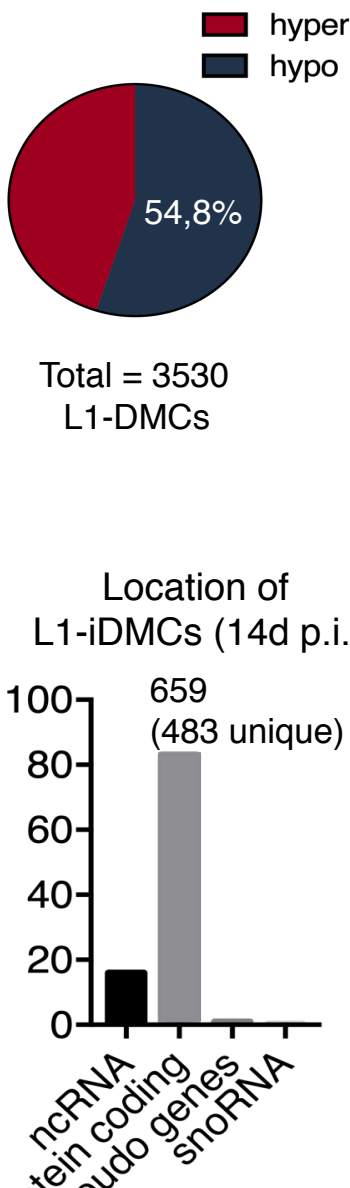

Ten most represented L1 families with L1-DMCs (14d p.i.)

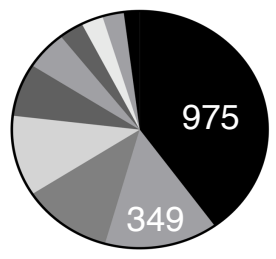

L1Md_F2

$\square$ L1Md_T

$\square$ L1Md_A

$\square$ L1Md_F3

$\square$ L1Md_F

$\square$ Lx8

$\square$ L1_Mus1

$\square$ L1_Mus3

Total $=3530$

L1-DMCs

E Ten most represented $L 1$ families with L1-iDMCs (14d p.i.)

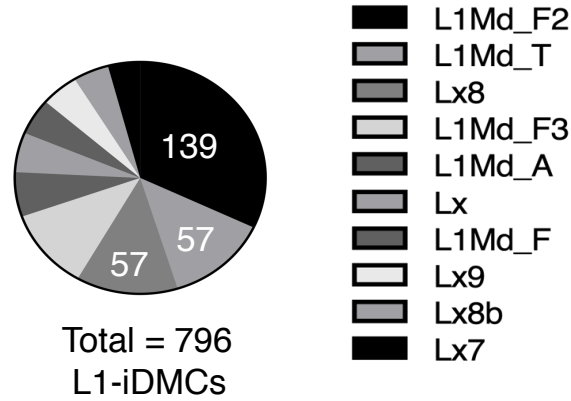

Fold enrichment

$\mathrm{H}$

DMC and DMRs overlapping with full-length LINE-1 (14d p.i.)

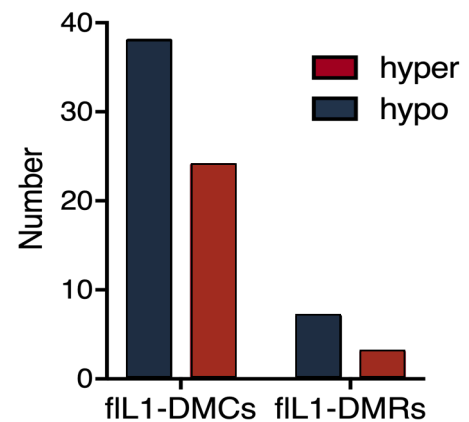

L1 A at 14d p.i.

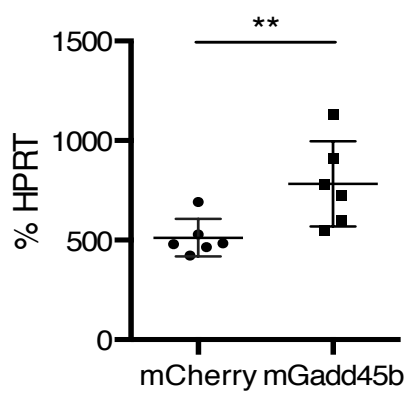

L1 Tf/Gf at 14d p.i.

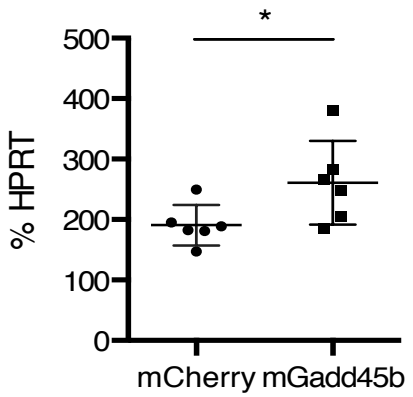

Fig. 5 
A

Satb1 at 14d p.i.

Setdb1 at 14d p.i

Dnmt3a at $14 d$ p.i.

TET3 at 14d p.i.

Park2 at 14d p.i
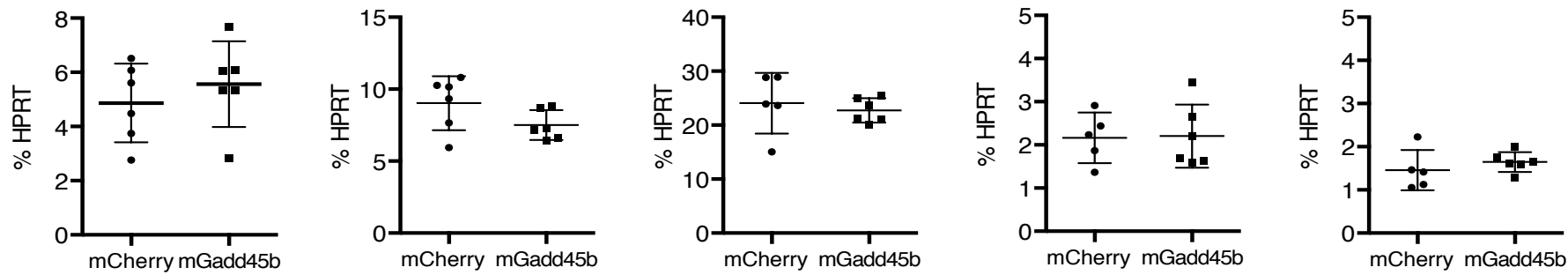

Satb1 at 90d p.i.

Setdb1 at 90d p.i.

Dnmt3a at 90d p.i.

TET3 at 90d p.i.
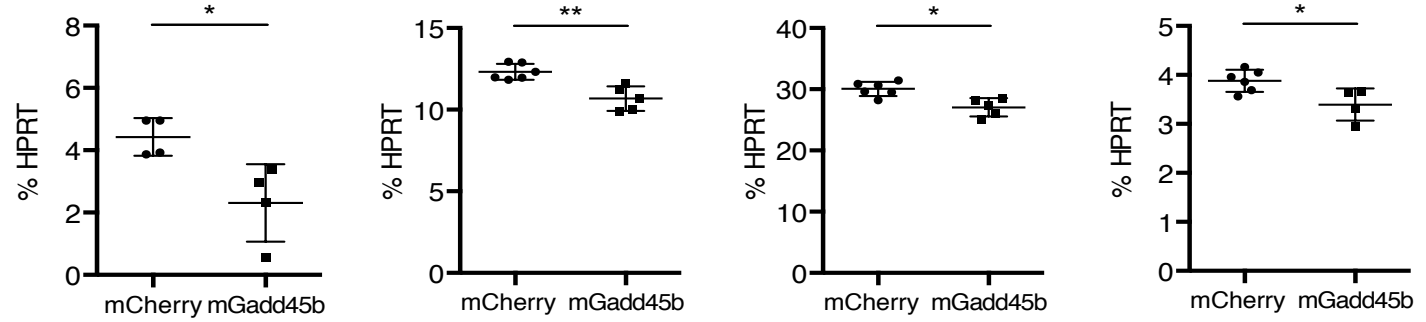

Park2 at 90d p.i

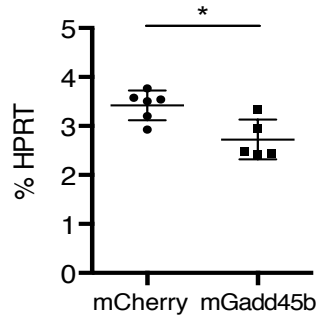

B

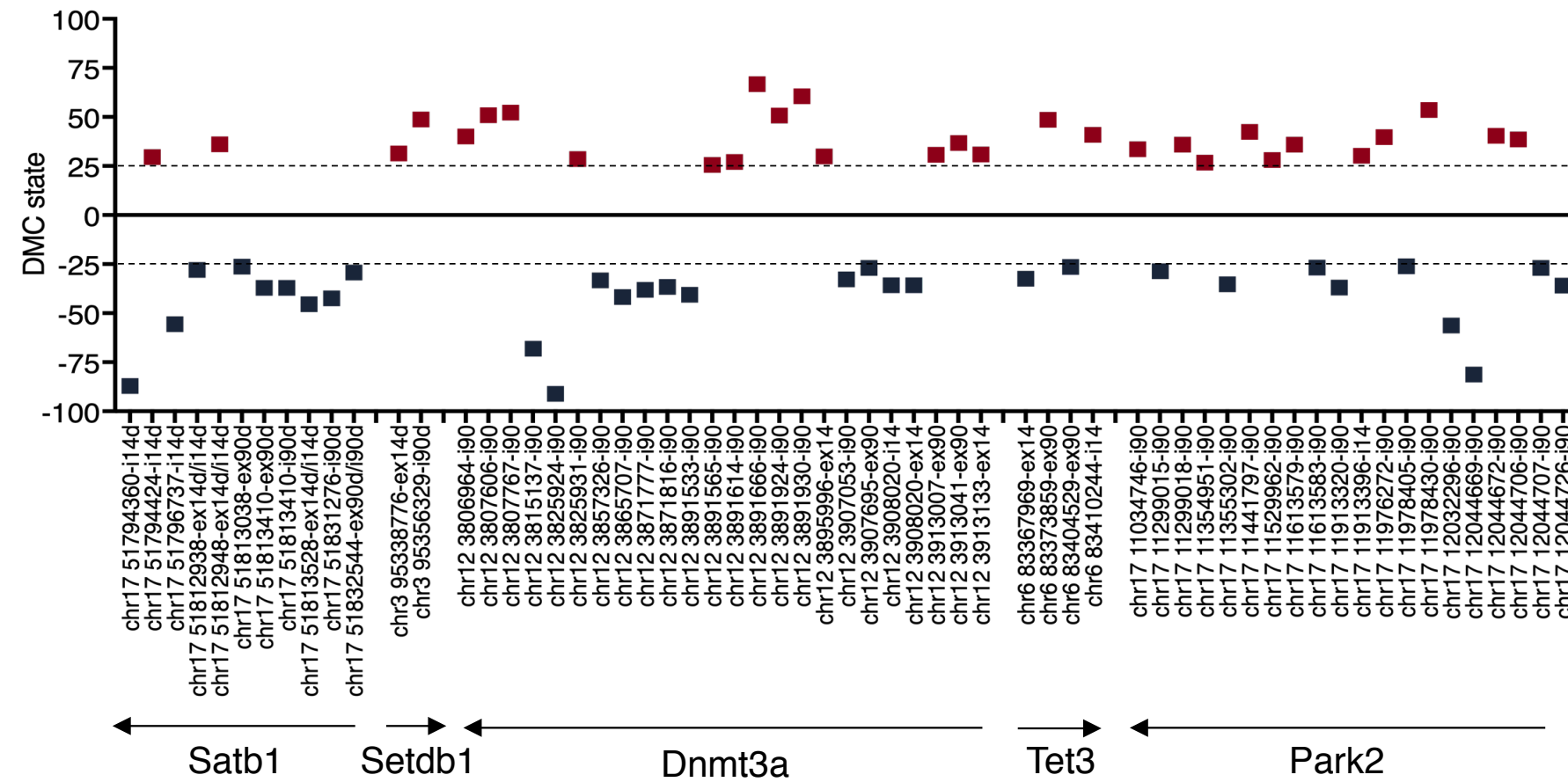

Fig. 6 


\begin{tabular}{|l|cccc|}
\hline Differential methylation analysis & 14d p.i. & & 90d p.i. \\
Gadd45b/ mCherry & DMCs & DMRs & DMCs & DMRs \\
\hline Total number & 76185 & 16702 & 76639 & 15685 \\
Hypomethylated (in \%) & $\mathbf{6 1 , 0 5}$ & $\mathbf{6 4 , 5 7}$ & $\mathbf{5 4 , 5 0}$ & $\mathbf{5 6 , 3 5}$ \\
Distance to CpG island (in \%) & & & & \\
\hline Islands & 15,34 & 1,50 & 16,03 & 1,30 \\
Shelves & 4,23 & 6,30 & 4,18 & 6,60 \\
Shores & 10,71 & 7,60 & 10,73 & 7,60 \\
Open sea & $\mathbf{6 9 , 7 3}$ & $\mathbf{8 4 , 6 0}$ & $\mathbf{6 9 , 0 6}$ & $\mathbf{8 4 , 6 0}$ \\
Association with genomic regions (in \%) & & & & \\
\hline Exons & $\mathbf{2 3 , 6 0}$ & $\mathbf{1 9 , 2 2}$ & $\mathbf{2 4 , 0 0}$ & $\mathbf{1 9 , 0 6}$ \\
Introns & $\mathbf{4 1 , 6 0}$ & $\mathbf{4 5 , 3 9}$ & $\mathbf{4 1 , 4 0}$ & $\mathbf{4 5 , 0 3}$ \\
Promoters & 12,50 & 8,57 & 12,80 & 9,03 \\
Intergenic & 22,30 & 26,83 & 21,90 & $\mathbf{2 6 , 8 8}$ \\
\hline
\end{tabular}

